Article

\title{
Effect of Addition of Natural Antioxidants on the Shelf-Life of "Chorizo", a Spanish Dry-Cured Sausage
}

\author{
Mirian Pateiro, Roberto Bermúdez, José Manuel Lorenzo and Daniel Franco * \\ Centro Tecnológico de la Carne de Galicia, Rúa Galicia No. 4, Parque Tecnológico de Galicia, \\ San Cibrao das Viñas, 32900 Ourense, Spain; E-Mails: mirianpateiro@ceteca.net (M.P.); \\ robertobermudez@ceteca.net (R.B.); jmlorenzo@ceteca.net (J.M.L.) \\ * Author to whom correspondence should be addressed; E-Mail: danielfranco@ceteca.net; \\ Tel.: +34-988-548-277; Fax: +34-988-548-276.
}

Academic Editors: Maria G. Miguel and João Rocha

Received: 30 October 2014 / Accepted: 6 January 2015 / Published: 14 January 2015

\begin{abstract}
The dose effect of the addition of natural antioxidants (tea, chestnut, grape seed and beer extracts) on physicochemical, microbiological changes and on oxidative stability of dry-cured "chorizo", as well as their effect during the storage under vacuum conditions was evaluated. Color parameters were significantly $(p<0.05)$ affected by the addition of antioxidants so that samples that contained antioxidants were more effective in maintaining color. The improving effects were dose-dependent with highest values with the dose of $50 \mathrm{mg} / \mathrm{kg}$ during ripening and depend on the extract during vacuum packaging. Addition of antioxidants decreased $(p<0.05)$ the oxidation, showing thiobarbituric acid reactive substances (TBARS) values below $0.4 \mathrm{mg}$ MDA $/ \mathrm{kg}$. Natural antioxidants matched or even improved the results obtained for butylated hydroxytoluene (BHT). Regarding texture profile analysis (TPA) analysis, hardness values significantly $(p<0.001)$ decreased with the addition of antioxidants, obtaining the lower results with the dose of $200 \mathrm{mg} / \mathrm{kg}$ both during ripening and vacuum packaging. Antioxidants reduced the counts of total viable counts (TVC), lactic acid bacteria (LAB), mold and yeast. Free fatty acid content during ripening and under vacuum conditions showed a gradual and significant $(p<0.05)$ release as a result of lipolysis. At the end of ripening, the addition of GRA 1000 protected chorizos from oxidative degradation.
\end{abstract}


Keywords: dry-cured chorizo; natural antioxidants; physicochemical parameters; microbial counts; lipid oxidation

\section{Introduction}

Agro-industries such as wineries and brewers have an economic relevance in the global market but also produce high quantities of wastes and by-products that could disrupt the environmental balance. There are many alternatives for reusing these materials, and their food utility has gained increasing interest. Their use as "natural" antioxidants could be one of the most efficient uses for these products. Their high content of phenolic compounds and their known antimicrobial power could lead to their use as substitutes for synthetic antioxidants.

For many years, the functional characteristics of many plant extracts have been evaluated because of their antioxidant and antimicrobial activity and their potential to replace synthetic antioxidants [1]. Grape (Vitis vinifera), green tea (Camellia sinensis) and chestnut (Castanea sativa) are of special interest due to their high content of phenolic compounds. Previous works reflect that grape seed extracts have antioxidant and antimicrobial activities in meat [2]; green tea was used to increase the shelf life of meat patties and pig liver pâté [3,4]; and the antioxidant activity of chestnut extract has also been investigated $[3,5]$.

"Chorizo" is a typical dry fermented sausage from Spain. During the manufacturing process of chorizo, microbiological, chemical and physicochemical changes take place. In particular dehydration, fermentation of carbohydrates and acidification, development of color, lipolysis and fat autooxidation and proteolysis takes place [6]. Therefore, the use of antioxidants during processing aims to delay oxidation [3], allowing increase the shelf life of the product. Industries generally used synthetic antioxidants to control this process, such as butylated hydroxyanisole (BHA), butylated hydroxytoluene (BHT) and tert-butylhydroquinone (THBQ). However, the use of these synthetic compounds has been linked to health risks (carcinogenic potential) and current research tends for their replacement by natural antioxidants [5]. Thereby, increasing interest in natural antioxidants and a search for naturally occurring compounds with antioxidant activity has increased dramatically [7].

For the conservation and to extent the shelf life of the product, vacuum-packaging under refrigeration together with the use of natural antioxidants could be used to prevent major changes during storage, especially removing oxygen, which is the main cause of food oxidation [8]. To our knowledge, not many studies regarding the effect of natural antioxidants on the oxidation stability of dry ripened sausage "chorizo" were found in the related literature [5]. In addition, not much data about the dose to be used of natural antioxidants is available, only on rosemary and tea extracts in sausages and patties, respectively $[9,10]$. Therefore, the aim of this study was to evaluate the dose effect of the addition of natural antioxidants (tea, chestnut, grape seed and beer extracts) on physicochemical, microbiological changes and on oxidative stability of dry-cured "chorizo", comparing their effect with a synthetic antioxidant (BHT), as well as knowing the effect of these natural extracts during the storage under vacuum conditions. 


\section{Experimental Section}

\subsection{Extraction of Natural Antioxidants}

Grape seed extract (GRA) and chestnut extract (CHE) were prepared as previously was indicated in Lorenzo et al. [5], while the extraction of green tea extract (TEA) was carried out as described in Lorenzo et al. [3]. Beer residue was provided by Hijos de Rivera S.A. (A Coruña, Spain). This residue was used as source of polyphenolic compounds. This suspension residue comes from process of boiling of the must, where the temperature is maintained at $102{ }^{\circ} \mathrm{C}$ for $90 \mathrm{~min}$. The objective of boiling is to obtain the necessary density, evaporating the spare water; the sterilizing the must and extracting and dissolving the wanted elements of hops. Lots of $4 \mathrm{~L}$ of this residue was transferred to XAD-16 amberlite column (Sigma-Aldrich, Spain). A glass column (7 cm $\varnothing$ in $\times 40 \mathrm{~cm}$ height) filled with XAD-16 amberlite was equilibrating with distillate water to separate polyphenolic compounds. Four liters of distillate water was poured on the column to remove impurities; later, three liters of ethanol was used to elute polyphenols. This volume was evaporated until $200 \mathrm{~mL}$ (or until ethanol was completely removed) remained. Subsequently, the residue was lyophilized using a freeze-dryer (Kinetics EZ-Dryer, Stone Ridge, NY, USA). This lyophilized extract (raw extract of beer by-product) rendered $9.41 \pm 34.0 \mathrm{~g} / \mathrm{L}$. This extract was subsequently used for the evaluation of the antioxidant capacity.

\subsection{Determination of Antioxidant Capacity}

\subsubsection{Determination of Total Phenolic Content}

The total phenolic content was determined using the Folin-Ciocalteu Reagent (FCR) with gallic acid as a standard. Readings were performed at $765 \mathrm{~nm}$ and were compared with a standard curve of gallic acid, being the total phenolic content expressed as mg of gallic acid equivalent per $\mathrm{g}$ of freeze dried solid (mg GAE/g). Analyses were performed in triplicate.

\subsubsection{Trolox Equivalent Antioxidant Capacity (TEAC)}

This assay is based on the scavenging of ABTS radical (2,2-azinobis-(3-ethyl-benzothiazoline-6sulphonate)), observed as a decolorization of blue-green color at $734 \mathrm{~nm}$. The radical scavenging capacity was compared with that of Trolox and results were expressed as $g$ of Trolox equivalent per $g$ of freeze dried solid.

\subsection{3. $\beta$-Carotene Bleaching Assay}

The $\beta$-carotene $(\beta C)$ bleaching assay described by Marco [11] was modified for use with microplates. Absorbance readings $(470 \mathrm{~nm})$ were taken at regular intervals in a ThermoFisher Scientific microplate reader until $\beta$-carotene was decolored (about $3 \mathrm{~h}$ ). The antioxidant activity coefficient (AAC) and $\mathrm{EC}_{50}$ value $(\mathrm{g} / \mathrm{L})$ were calculated as previously described in Lorenzo et al. [3] for each antioxidant extract. 


\subsection{4. $\alpha, \alpha$-Diphenyl- $\beta$-Picrylhydrazyl (DPPH) Radical Scavenging Activity}

The antioxidant activity was determined with DPPH as a free radical, using microplate. Antioxidant solutions $(10 \mu \mathrm{L})$ were added in triplicate to $200 \mu \mathrm{L}$ of a $60 \mu \mathrm{M}$ solution of DPPH in $70 \%$ ethanol. The decrease in absorbance was followed at $515 \mathrm{~nm}$ every 5 min until the reaction reached a plateau (about $2 \mathrm{~h}$ ). The $\mathrm{EC}_{50}$ and BHT equivalent activity were calculated as explained above.

\subsection{Manufacture of Dry-Cured Sausages}

Four batches (20 units per batch, 3 per ripening time) of dry-cured sausage "chorizo": Control $(\mathrm{CON}), \mathrm{BHT}$, grape seed (GRA), chestnut (CHE), green tea (TEA) and beer extracts (BER) were manufactured in the pilot plant of the Meat Technology Center of Galicia. Sausages were manufactured using the primal cuts of shoulder (85\%) and pork back fat (15\%) from Celta pig breed. The lean and the pork back fat were ground through a $6 \mathrm{~mm}$ diameter mincing plate in a refrigerated mincer machine (La Minerva, Bologna, Italy). Mixture was vacuum minced in a vacuum mincer machine (Fuerpla, Valencia, Spain) for 3 min with $5 \mathrm{~g} / \mathrm{kg}$ of $\mathrm{NaCl}, 20 \mathrm{~g} / \mathrm{kg}$ of sweet paprika, $3 \mathrm{~g} / \mathrm{kg}$ of spicy paprika, $0.5 \mathrm{~g} / \mathrm{kg}$ of garlic and $200 \mathrm{mg} / \mathrm{kg}$ of BHT for BHT batch, $0.05-0.2-1 \mathrm{~g} / \mathrm{kg}$ of natural extracts. No starter culture was added. The meat mixture was maintained at $3-5{ }^{\circ} \mathrm{C}$ for $24 \mathrm{~h}$ and then was stuffed into pig gut (diameter $32-34 \mathrm{~mm}$ ) to obtain an average final sausage weight of $150 \mathrm{~g}$. After stuffing, the sausages were conditioned for two days at $7{ }^{\circ} \mathrm{C}$ and $85 \%$ of relative humidity. The sausages were transferred to a drying-ripening chamber where they were kept for 48 days at $12{ }^{\circ} \mathrm{C}$ and $75 \%-80 \%$ of relative humidity. Below the samples were packed under vacuum conditions five months at $4{ }^{\circ} \mathrm{C}$. Analyses were carried out at $0,4,19$ and 48 days of ripening time and at 2, 4 and 7 months of vacuum-packaged. The studied parameters were determined in duplicate for every sampling point.

\subsection{Determination of $\mathrm{pH}$, Moisture Content, Water Activity and Color Parameters}

The $\mathrm{pH}$ of samples was measured using a pH-meter (HI 99163, Hanna Instruments, Eibar, Spain) equipped with a glass probe for penetration. Moisture percentage was determined by oven drying (Memmert UFP 600, Schwabach, Germany) at $105{ }^{\circ} \mathrm{C}$ until constant weight [12], and calculated as sample (5 g) weight loss. Water activity was determined using a Fast-lab (Gbx, Romans sur Isére, Cédex, France) water activity meter, previously calibrated with sodium chloride. A portable colorimeter (Konica Minolta CM-600d, Osaka, Japan) with pulsed xenon arc lamp, $0^{\circ}$ viewing angle geometry and $8 \mathrm{~mm}$ aperture size, was used to estimate meat color in the CIELAB space: lightness, (L*); redness, $\left(a^{*}\right)$; yellowness, $\left(b^{*}\right)$. Each sausage piece was cut $(2 \mathrm{~cm})$ and the color of the slices was measured three times for each analytical point.

\subsection{Determination of Lipid Oxidation}

Lipid stability was evaluated through TBARS index according to the method proposed by Targladis et al. [13]. Briefly, the dry-cured sausage sample (10 g) was dispersed in distilled water $(50 \mathrm{~mL})$ and homogenized in an Ultra-Turrax (Ika T25 basic, Staufen, Germany) for $2 \mathrm{~min}$. The homogenate was carried to a distillation system with $\mathrm{HCl} 4 \mathrm{~N}(2.5 \mathrm{~mL})$ and distilled water $(47.5 \mathrm{~mL})$ until recover $50 \mathrm{~mL}$ of distilled. The filtrate $(5 \mathrm{~mL})$ was reacted with a $0.02 \mathrm{M}$ thiobarbituric acid (TBA) solution $(5 \mathrm{~mL})$ and 
incubated in a water bath at $96{ }^{\circ} \mathrm{C}$ for $40 \mathrm{~min}$. The absorbance was measured at $538 \mathrm{~nm}$. Thiobarbituric acid reactive substances (TBARS) values were calculated from a standard curve of malonaldehyde with 1,1,3,3-tetraethoxipropane (TEP) and expressed as mg MDA/kg sample.

\subsection{Determination of Texture Profile Analysis}

Texture profile analysis (TPA) was measured by compressing to $50 \%$ with a compression probe of $19.85 \mathrm{~cm}^{2}$ of surface contact in seven dry-cured sausage slices of $2 \mathrm{~cm}$ using a texture analyzer (TA.XTplus, Stable Micro Systems, Vienna Court, UK). Force-time curves were recorded at a crosshead speed of $1 \mathrm{~mm} / \mathrm{s}$. Hardness $(\mathrm{kg})$, cohesiveness, springiness $(\mathrm{mm})$, gumminess $(\mathrm{kg})$ and chewiness $(\mathrm{kg} \times \mathrm{mm})$ were obtained. These parameters were obtained using the available computer software (Texture Exponent 32 (version 1.0.0.68), Stable Micro Systems, Vienna Court, UK).

\subsection{Analysis of Free Fatty Acid Content}

Total intramuscular lipids were extracted from $5 \mathrm{~g}$ of each minced sausage sample, according to Folch et al. [14] procedure. Free fatty acids were separated from fifty milligrams of the extracted lipids using aminopropyl $\left(\mathrm{NH}_{2}\right)$ mini-columns as described by García-Regueiro et al. [15]. This fraction was transesterified with a solution of boron trifluoride (14\%) in methanol, according to Carreau and Dubacq [16] and the FAMEs were stored at $-80{ }^{\circ} \mathrm{C}$ until chromatographic analysis. Separation and quantification of FAMEs was determined following Lorenzo and Franco [17].

\subsection{Microbial Analysis}

For microbiological analysis, a $10 \mathrm{~g}$ sample of dry-cured sausage was aseptically weighted in a sterile plastic bag. Subsequently samples were homogenized with $90 \mathrm{~mL}$ of a sterile solution of $0.1 \%(\mathrm{w} / \mathrm{v})$ peptone water (Oxoid, Unipath, Basingstoke, UK), containing $0.85 \% \mathrm{NaCl}$ and $1 \%$ Tween 80 as emulsifier, for $2 \mathrm{~min}$ at $20-25{ }^{\circ} \mathrm{C}$ in a Masticator blender (IUL Instruments, Barcelona, Spain), thus making a $1 / 10$ dilution. Serial 10 -fold dilutions were prepared by mixing $1 \mathrm{~mL}$ of the previous dilution with $9 \mathrm{~mL}$ of $0.1 \%(\mathrm{w} / \mathrm{v})$ sterile peptone water. Total viable counts (TVC) were enumerated in Plate Count Agar (PCA; Oxoid, Unipath Ltd., Basingstoke, UK) and incubated at $30^{\circ} \mathrm{C}$ for $48 \mathrm{~h}$; lactic acid bacteria (BAL) were determined on the Man Rogosa Sharpe medium Agar (Oxoid, Unipath Ltd., Basingstoke, UK) (pH 5.6) after an incubation at $30{ }^{\circ} \mathrm{C}$ for 5 days. After incubation, plates with 30-300 colonies were counted. The microbiological data were transformed into logarithms of the number of colony forming units (CFU/g).

\subsection{Statistical Analysis}

For the statistical analysis of the results, an analysis of variance (ANOVA) of one way using SPSS package (SPSS 19.0, Chicago, IL, USA) was performed for all variables considered in the study. The least squares mean (LSM) were separated using Duncan's $t$-test. All statistical test of LSM were performed for a significance level $p<0.05$. 


\section{Results and Discussion}

\subsection{Antioxidant Activity of the Extracts}

GRA and TEA extracts showed the highest polyphenol content, mainly flavonoids and flavan-3-ols, which antioxidant activity has been demonstrated $[2,18]$. The major compounds found in TEA extracts was catechin, epicatechin, cinnamic acids and sugar-linked flavonols [19], while GRA extracts contained benzoic acids, monomer flavan-3-ols and oligomeric procyanidins [18]. The higher activity found in GRA extracts could be associated to its resveratrol content [20]. Regarding polyphenols in CHE and BER extracts, their concentration were significantly lower than the aforementioned natural extracts (28.9 and 89.0 vs. 373.0 and $390.0 \mathrm{mg} \mathrm{GAE} / \mathrm{g}$ extract for BER and CHE vs. GRA and TEA extracts, respectively).

TEAC, DPPH and $\beta$-carotene were used to assess in vitro antioxidant activity of the natural extracts. These methods were directly related to polyphenol contents [4]. Therefore, GRA and TEA extracts showed the highest activities in these methods. In the case of TEAC, the aforementioned extracts displayed values 10 and 15-fold higher Trolox equivalent antioxidant capacity than CHE extract ( 0.27 vs. 2.93 and 4.06 g Trolox/g extract for CHE, GRA and TEA, respectively) and 20 and 40-fold higher than BER extract ( $0.09 \mathrm{~g}$ Trolox/g extract).

The scavenging activity found on DPPH radical showed the higher antioxidant power of BHT standard, followed by GRA and TEA extracts (1.80 and $2.18 \mathrm{~g}$ equivalent BHT/g extract, respectively). The values provided by BER and CHE were almost 4 and 8 -fold lower than the aforementioned extracts ( 0.25 and $0.48 \mathrm{~g}$ equivalent $\mathrm{BHT} / \mathrm{g}$ extract, respectively). The $\mathrm{EC}_{50}$ values obtained showed the same behavior, the powerful antioxidant activity of TEA and GRA $(0.12$ and $0.16 \mathrm{~g}$ extract/L, respectively) vs. BER and CHE extracts (data not shown).

$\beta$-carotene bleaching assay of the natural extracts showed similar activity values for CHE and TEA ( 0.53 and $0.69 \mathrm{~g}$ equivalent BHT/g extract, respectively), although GRA were the most active ( $1.28 \mathrm{~g}$ equivalent BHT/g extract) and BER the least active ( $0.25 \mathrm{~g}$ equivalent BHT/g extract). The EC $\mathrm{E}_{50}$ values obtained displayed rather similar activity values for all the extracts (less than $0.10 \mathrm{~g}$ extract/L).

\subsection{Effect of Antioxidants on Physicochemical Parameters during the Manufacturing Process and Vacuum Packaging}

Changes occurred in $\mathrm{pH}$, moisture content and water activity $\left(\mathrm{a}_{\mathrm{w}}\right)$ during the manufacturing process and vacuum-packing are given in Figure 1. Ripening time had a significant effect $(p<0.01)$ on $\mathrm{pH}$ values. During the first 19 days of ripening, $\mathrm{pH}$ values decreased from 5.62 to approximately 5.43 due to the production of lactic acid as a result of carbohydrate breakdown during fermentation [21] and the following increase can be produced by the liberation of peptides, amino acids and ammonia from proteolytic reactions [17]. Although at the end of ripening $\mathrm{pH}$ values of sausages were not affected $(p>0.05)$ by the addition of antioxidants, the highest $\mathrm{pH}$ values were observed in $\mathrm{CHE}_{50}$ and GRA50, followed by $\mathrm{CON}$ and BER50. These $\mathrm{pH}$ values were similar to those found in other varieties of sausages [22,23]. Regarding the evolution during vacuum packaging, there are not many studies that evaluate the influence of antioxidants on physicochemical parameters of dry-cured sausages. The trend is to continue growing slightly until 120 days to decreasing until the end of storage. The exception to 
this behavior is found in CON and TEA samples, which $\mathrm{pH}$ values continued increasing until the end of storage. Regarding dose effect, only TEA and GRA extracts presented significant $(p<0.05)$ differences on $\mathrm{pH}$ during ripening time and vacuum storage.

Moisture content was significantly $(p<0.01)$ affected by ripening time and addition of antioxidants, decreasing during the drying period as a result of moisture loss at high ripening temperature and low percentage of relative humidity (Figure 1). CON was the sample that presented the lowest value at the end of this stage with mean values of $20.6 \%$, while samples manufactured with antioxidants presented higher values, in all cases above $22.0 \%$. The moisture content and water activity followed similar behaviors because are variables that are intrinsically linked. As occurred with water content, the trend of water activity was to decrease over the time, obtaining significant differences during ripening time $(p \leq 0.001)$. As can be observed in Figure 1, two steps could be distinguished in its evolution. The first one represented a sharp decline in the values during ripening stage and the second one stabilization during the vacuum packaging until the end of the storage. No significant differences $(p>0.05)$ were observed in moisture and water activity among extracts depending on dose effect during ripening time and vacuum packaging.

Color parameters of the chorizo were significantly $(p<0.05)$ affected by ripening time and addition of antioxidants (Table 1). Regarding redness $\left(\mathrm{a}^{*}\right)$, the trend was to decrease over time. Thereby, a significant $(p<0.01)$ color loss was observed during ripening time with values ranged between 27.6 and 21.2. This behavior could be due to the partial or total denaturation of nitrosomyoglobin caused by the production of lactic acid. Furthermore, a significant effect $(p<0.05)$ was also observed on redness with the use of antioxidants. Antioxidants were more effective in maintaining color, with higher values of redness in the samples manufactured with antioxidants. In this sense, samples manufactured with GRA200, $\mathrm{CHE}_{50}, \mathrm{CHE}_{200}$ and BHT presented higher a* values compared to CON batch; so that the addition of natural extracts improved the color stability, showing even better results than those showed by BHT.

During vacuum packaging, a slightly increase was observed in redness values. A similar behavior during packaging under vacuum was reported by Liaros et al. [24]. As happened during ripening, significant differences $(p<0.001)$ were also found with the addition of antioxidants. Regarding dose effect, significant differences $(p<0.01)$ were found for this color parameter in samples that contained TEA, CHE and GRA in their composition. During ripening, the highest values were obtained in the samples that containing a dose of $50 \mathrm{mg} / \mathrm{kg}$ in their composition, while vacuum packaging the dose more effective depends on the used extract. In this regards, Jayawardana et al. [25] showed the capacity of natural extracts to hold the color on pork sausages. As in the present study, the improving effects of extracts were concentration-dependent [1]. In this case, with the exception of samples that contain BER extract, a decrease of redness occurs at increasing levels of antioxidants. 

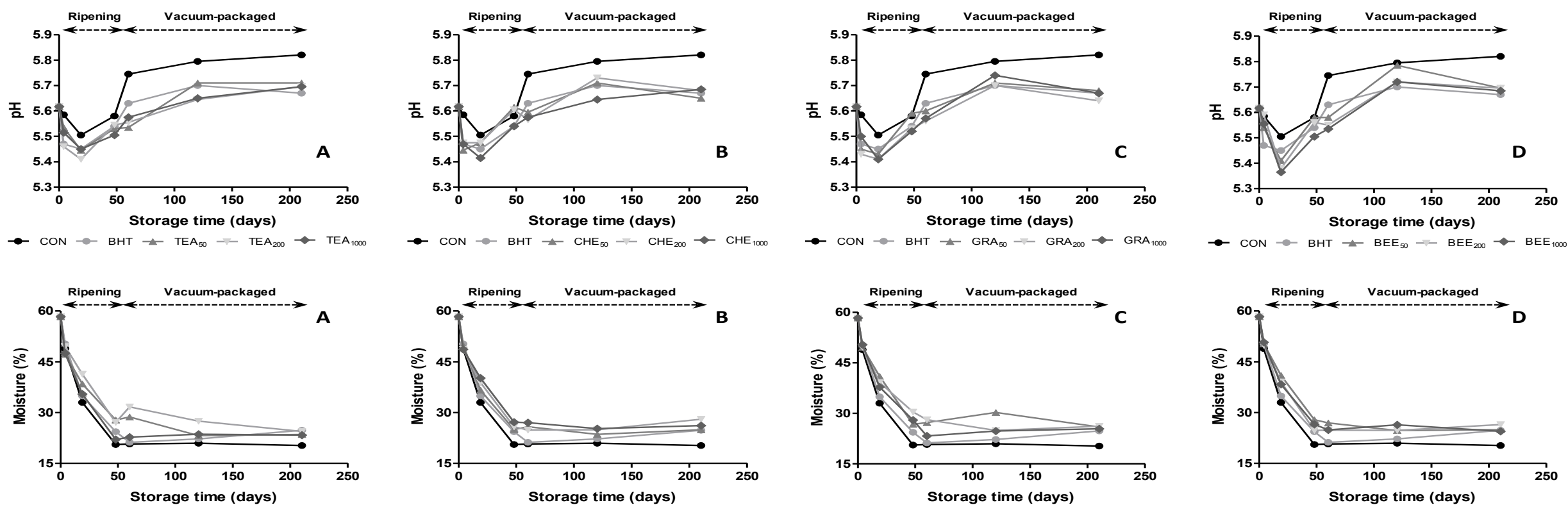

$\rightarrow$ CON $\rightarrow$ BHT $\rightarrow \mathrm{TEA}_{50} \rightarrow \mathrm{TEA}_{200} \rightarrow \mathrm{TEA}_{100}$
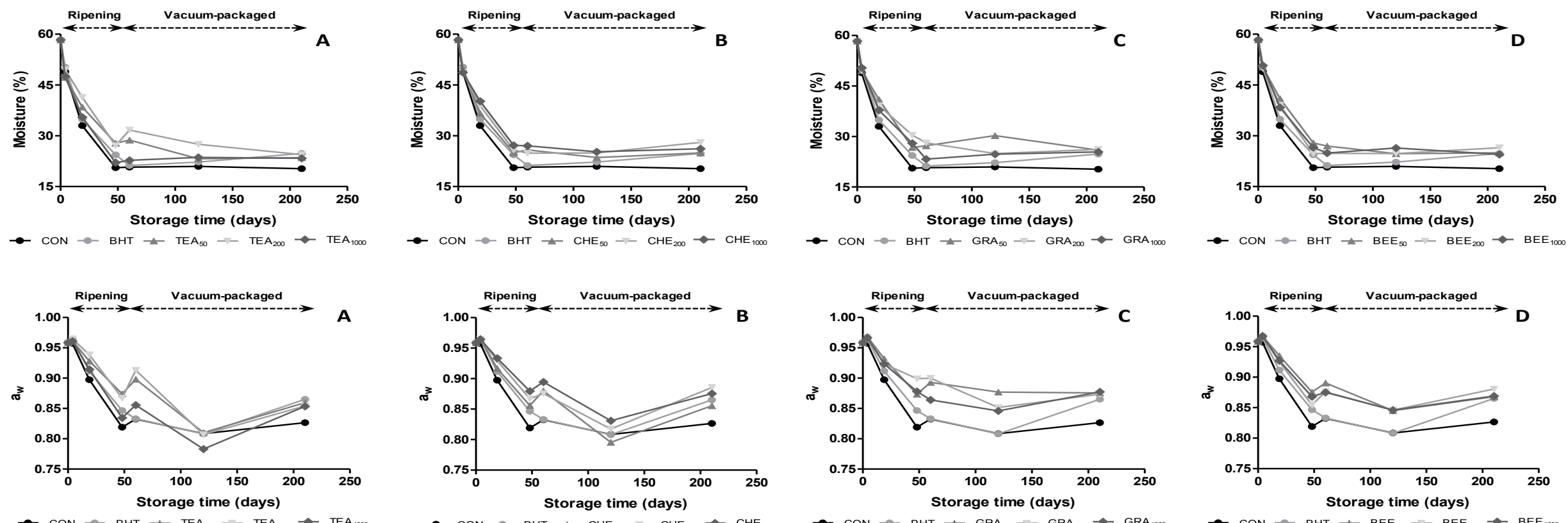

Figure 1. Evolution of $\mathrm{pH}$ values, moisture content and water activity in dry-cures sausages treated with butylated hydroxytoluene (BHT) and natural antioxidants during ripening and vacuum-packaged storage. 
Table 1. Evolution of color parameters of "chorizo" treated with BHT and natural antioxidants during ripening and vacuum-packaging.

\begin{tabular}{|c|c|c|c|c|c|c|c|c|c|c|c|c|c|c|c|c|c|}
\hline & \multirow{2}{*}{ Days } & \multirow{2}{*}{ CON } & \multirow{2}{*}{$\begin{array}{c}\text { BHT } \\
200 \\
\end{array}$} & \multicolumn{3}{|c|}{ TEA } & \multicolumn{3}{|c|}{ CHE } & \multicolumn{3}{|c|}{ GRA } & \multicolumn{3}{|c|}{ BER } & \multirow{2}{*}{$p$-Value } & \multirow{2}{*}{ SEM } \\
\hline & & & & 50 & 200 & 1000 & 50 & 200 & 1000 & 50 & 200 & 1000 & 50 & 200 & 1000 & & \\
\hline \multirow{8}{*}{$L^{*}$} & 4 & $43.30^{\mathrm{e}}$ & $44.86^{\mathrm{e}}$ & $43.79^{\mathrm{d}}$ & $43.04^{c}$ & $42.52^{\mathrm{c}}$ & $43.94^{\mathrm{b}}$ & $42.51^{\mathrm{b}}$ & $43.22^{\mathrm{c}}$ & $44.61^{\mathrm{d}}$ & $45.09^{\mathrm{d}}$ & $42.55^{\mathrm{c}}$ & $42.34^{\mathrm{c}}$ & $42.33^{\mathrm{c}}$ & $43.40^{\mathrm{d}}$ & 0.186 & 0.23 \\
\hline & 19 & $34.30^{\mathrm{d}, 1}$ & $40.05^{\mathrm{d}, 3}$ & $41.88^{\mathrm{d}, 3,4}$ & $43.19^{\mathrm{c}, 4}$ & $41.16^{c, 3,34}$ & $42.49^{\mathrm{b}, 4}$ & $42.60^{\mathrm{b}, 4}$ & $41.62^{c, 3,3,4}$ & $42.58^{\mathrm{c}, \mathrm{d}, 4}$ & $41.85^{\mathrm{d}, 3,4}$ & $35.70^{b, 1,2}$ & $36.49^{\mathrm{b}, 2}$ & $37.11^{b, 2}$ & $37.21^{c, 2}$ & 0.000 & 0.58 \\
\hline & 48 & $30.46^{\mathrm{c}, 1}$ & $35.34^{c, 2-4}$ & $38.98^{c, 4}$ & $36.54^{6,3,4}$ & $32.03^{a, b, 1,2}$ & $35.51^{\mathrm{a}, 2-4}$ & $34.88^{\mathrm{a}, 2,3}$ & $36.24^{b, 3,4}$ & $36.96^{a \mathrm{ab}, 3,4}$ & $37.36^{\mathrm{c}, 34}$ & $34.70^{b, 2,3}$ & $33.47^{\mathrm{a}, 1-3}$ & $32.13^{\mathrm{a}, 1,2}$ & $34.56^{b, 2,3}$ & 0.008 & 0.49 \\
\hline & 60 & $29.00^{b, c, 1,2}$ & $31.68^{a, 3,4}$ & $38.32^{c, 8}$ & $39.23^{b, c, 8}$ & $33.59^{\mathrm{b}, 4,5}$ & $34.48^{a, 5,6}$ & $33.57^{a, 4,5}$ & $34.60^{\mathrm{a}, \mathrm{b}, 5,6}$ & $37.55 \mathrm{ab}, 7,8$ & 35.94 b,c,6,7 & $28.39^{\mathrm{a}, 1}$ & $31.81^{a, 3,4}$ & $31.08^{a, 2,3}$ & $29.24^{a, 1,2}$ & 0.000 & 0.66 \\
\hline & 120 & $28.63^{\mathrm{b}, 1}$ & $34.49^{\mathrm{b}, \mathrm{c}, 3-5}$ & $35.58^{\mathrm{b}, 5,6}$ & $38.40^{\mathrm{b}, 6,7}$ & $32.93^{b, 2-5}$ & $33.54^{a, 2-5}$ & $35.06^{a, 4,5}$ & $35.01^{\mathrm{a}, \mathrm{b}, \mathrm{b}, \mathrm{s}}$ & $39.68^{b, c, 7}$ & $33.29^{\mathrm{a}, \mathrm{b}, 2-5}$ & $30.81^{\mathrm{a}, 1,2}$ & $31.09^{a, 1-3}$ & $31.64^{2,1-4}$ & $31.89^{a, 1-4}$ & 0.000 & 0.59 \\
\hline & 210 & $26.80^{\mathrm{a}, 1}$ & $32.84^{\mathrm{a}, \mathrm{b}, 2,3}$ & $31.85^{a, 2,3}$ & $31.41^{\mathrm{a}, 2}$ & $30.38^{\mathrm{a}, 2}$ & $31.78^{a, 2,3}$ & $34.86^{\mathrm{a}, 3}$ & $32.34^{a, 2,3}$ & $34.71^{\mathrm{a}, 3}$ & $30.52^{\mathrm{a}, 2}$ & $30.24^{\mathrm{a}, 2}$ & $31.08^{a, 2}$ & $32.04^{\mathrm{a}, 2,3}$ & $30.04^{\mathrm{a}, 2}$ & 0.003 & 0.41 \\
\hline & $\begin{array}{c}p- \\
\text { value }\end{array}$ & 0.000 & 0.000 & 0.000 & 0.005 & 0.000 & 0.001 & 0.005 & 0.001 & 0.001 & 0.001 & 0.000 & 0.000 & 0.000 & 0.000 & & \\
\hline & SEM & 1.82 & 1.39 & 1.20 & 1.27 & 1.41 & 1.43 & 1.20 & 1.22 & 1.05 & 1.52 & 1.43 & 1.24 & 1.24 & 1.48 & & \\
\hline \multirow{8}{*}{$a^{*}$} & 4 & $26.17^{\mathrm{c}, \mathrm{d} 2-2-5}$ & $27.30^{\mathrm{a}, \mathrm{b}, 5}$ & $26.57^{\mathrm{a}, 3-5}$ & $25.82^{a, 1-5}$ & $24.90^{\mathrm{b}, \mathrm{c}, 1,2}$ & $26.07^{\mathrm{a}, 2345}$ & $25.44^{\mathrm{a}, 1-4}$ & $24.43^{\mathrm{a}, \mathrm{b}, 1}$ & $26.60^{a, 3-5}$ & $26.94^{a, 4,5}$ & $24.53^{1}$ & $26.24^{2-5}$ & $25.26^{1-3}$ & $26.17^{2-5}$ & 0.007 & 0.18 \\
\hline & 19 & $23.58^{a-c, 1}$ & $28.43^{b, c, 3,34}$ & $29.43^{b, c, 4,5}$ & $29.21^{b, 4,5}$ & $26.90^{c, 2,3}$ & $29.15^{\mathrm{b}, 4,5}$ & $29.09^{\mathrm{b}, 4,4,5}$ & $27.02^{b, c, 2,3}$ & $30.51^{b, c, 5}$ & $29.80^{\mathrm{b}, 4,5}$ & $24.37^{1}$ & $26.97^{2,3}$ & $26.80^{2,3}$ & $26.69^{2}$ & 0.000 & 0.39 \\
\hline & 48 & $21.20^{\mathrm{a}, 1}$ & $25.44^{\mathrm{a}, \mathrm{b}, 2-6}$ & $28.18^{\mathrm{a}, \mathrm{b}, \mathrm{b}}$ & $24.82^{\mathrm{a}, 2-6}$ & $22.23^{\mathrm{a}, \mathrm{b}, \mathrm{l}, \mathrm{2}}$ & $25.93^{a, 3-6}$ & $25.46^{a, 2-6}$ & $24.27^{\mathrm{a}, \mathrm{b}, 1-5}$ & $27.67^{\mathrm{abb}, 5,6}$ & $27.28^{a, 4-6}$ & $23.71^{1-4}$ & $24.87^{2-6}$ & $22.73^{1-3}$ & $24.44^{1-5}$ & 0.010 & 0.43 \\
\hline & 60 & $22.61^{a, b, 1}$ & $26.57^{a, b, 3}$ & $31.88^{\mathrm{c}, 5}$ & $32.25^{\mathrm{c}, 5}$ & $26.06^{c, 2,3}$ & $26.77^{\mathrm{a}, \mathrm{b}, 3}$ & $26.79^{a-c, 3}$ & $25.54^{\mathrm{a}-\mathrm{c}, 2,3}$ & $29.59^{a-c, 4}$ & $29.53^{b, 4}$ & $23.02^{1}$ & $25.89^{2,3}$ & $26.97^{3}$ & $24.22^{1,2}$ & 0.000 & 0.56 \\
\hline & 120 & $23.94^{\mathrm{acc}, 1}$ & $30.42^{\mathrm{c}, 4,5}$ & $30.73^{b, c, 45}$ & $32.33^{c, 5}$ & $26.07^{\mathrm{c}, 1-3}$ & $29.16^{\mathrm{b}, 3-5}$ & $29.90^{\mathrm{c}, 4,5}$ & $28.07^{\mathrm{c}, 2-4}$ & $31.91^{c, 5}$ & $26.06^{\mathrm{a}, 1-3}$ & $23.11^{1}$ & $24.60^{1}$ & $24.88^{1,2}$ & $25.23^{1,2}$ & 0.000 & 0.61 \\
\hline & 210 & $24.93^{b-d}$ & $24.93^{\mathrm{a}}$ & $26.17^{\mathrm{a}}$ & $25.07^{\mathrm{a}}$ & $20.85^{\mathrm{a}}$ & $25.00^{\mathrm{a}}$ & $25.85^{\mathrm{a}, \mathrm{b}}$ & $22.86^{\mathrm{a}}$ & $27.83^{a, b}$ & $26.23^{a}$ & 22.80 & 23.59 & 25.12 & 25.25 & 0.066 & 0.39 \\
\hline & $\begin{array}{c}p- \\
\text { value }\end{array}$ & 0.007 & 0.030 & 0.010 & 0.001 & 0.016 & 0.033 & 0.051 & 0.046 & 0.026 & 0.005 & 0.805 & 0.229 & 0.126 & 0.117 & & \\
\hline & SEM & 0.59 & 0.62 & 0.67 & 0.99 & 0.72 & 0.54 & 0.60 & 0.59 & 0.60 & 0.47 & 0.39 & 0.43 & 0.51 & 0.32 & & \\
\hline
\end{tabular}


Table 1. Cont.

\begin{tabular}{|c|c|c|c|c|c|c|c|c|c|c|c|c|c|c|c|c|c|}
\hline & \multirow{2}{*}{ Days } & \multirow{2}{*}{ CON } & \multirow{2}{*}{$\begin{array}{c}\text { BHT } \\
200 \\
\end{array}$} & \multicolumn{3}{|c|}{ TEA } & \multicolumn{3}{|c|}{ CHE } & \multicolumn{3}{|c|}{ GRA } & \multicolumn{3}{|c|}{ BER } & \multirow{2}{*}{$p$-Value } & \multirow{2}{*}{ SEM } \\
\hline & & & & 50 & 200 & 1000 & 50 & 200 & 1000 & 50 & 200 & 1000 & 50 & 200 & 1000 & & \\
\hline \multirow{8}{*}{$b^{*}$} & 4 & $32.50^{\mathrm{c}}$ & $35.10^{\mathrm{e}}$ & $35.28^{c}$ & $33.95^{\mathrm{b}}$ & $33.93^{\mathrm{c}}$ & $34.11^{\mathrm{c}}$ & $34.22^{\mathrm{b}}$ & $32.84^{\mathrm{c}}$ & $35.85^{\mathrm{c}}$ & $37.01^{\mathrm{d}}$ & $33.15^{\mathrm{c}}$ & $34.04^{\mathrm{d}}$ & $33.13^{\circ}$ & $33.92^{d}$ & 0.249 & 0.30 \\
\hline & 19 & $21.59^{\mathrm{b}, 1}$ & $30.22^{\mathrm{d}, 3}$ & $30.91^{b, c, 3}$ & $33.06^{\mathrm{b}, 3}$ & $31.13^{\mathrm{c}, 3}$ & $32.40^{\mathrm{c}, 3}$ & $33.77^{\mathrm{b}, 3}$ & $31.89^{\mathrm{c}, 3}$ & $33.43^{\mathrm{c}, 3}$ & $32.99^{\mathrm{c}, 3}$ & $23.36^{\mathrm{b}, 1,2}$ & $25.22^{\mathrm{c}, 2}$ & $26.13^{b, 2}$ & $25.06^{\mathrm{c}, 2}$ & 0.000 & 0.80 \\
\hline & 48 & 19.62 a,b,1 & $23.71^{c, 1-4}$ & $30.73^{b, c, 5}$ & $23.01^{\mathrm{a}, 1-4}$ & $20.55^{b, 1,2}$ & $25.15^{b, 1-5}$ & $26.28^{\mathrm{a}, 2-5}$ & $25.73^{b, 2-5}$ & $28.50^{b, 4,5}$ & $27.52^{b, 3-5}$ & $24.02^{b, 1-4}$ & $22.31^{b, c, 1-3}$ & $21.86^{\mathrm{a}, 1-3}$ & $23.30^{b, c, 1-4}$ & 0.014 & 0.66 \\
\hline & 60 & $17.09^{\mathrm{a}, 1,2}$ & $20.17^{\mathrm{a}, \mathrm{b}, 2-4}$ & $27.84^{b, 7,8}$ & $30.03^{\mathrm{b}, 8}$ & $21.57^{\mathrm{b}, 3-5}$ & $22.32^{\mathrm{a}, \mathrm{b}, 46}$ & $21.64^{a, 3-5}$ & $21.88^{a, b, 3-5}$ & $25.28^{\mathrm{a}, \mathrm{b}, 6,7}$ & $24.10^{a, 5,6}$ & $16.69^{\mathrm{a}, 1}$ & $18.81^{\mathrm{a}, \mathrm{b}, \mathrm{l}-\mathrm{3}}$ & $19.97^{\mathrm{a}, 2-4}$ & $16.68^{\mathrm{a}, 1}$ & 0.000 & 0.77 \\
\hline & 120 & 17.82 a,b, & $23.45^{b, c, 2,3}$ & $26.36^{b, 3,4}$ & $28.91^{b, 4}$ & $22.37^{b, 1-3}$ & $23.58^{b, 2,3}$ & $26.30^{\mathrm{a}, 3,4}$ & $25.45^{b, 3,4}$ & $28.97^{\mathrm{b}, 4}$ & $21.45^{\mathrm{a}, 1-3}$ & $17.73^{\mathrm{a}, 1}$ & $19.28 \mathrm{ab}, 1,1,2$ & $20.23^{\mathrm{a}, 1,2}$ & $20.19^{a, b, 1,2}$ & 0.001 & 0.76 \\
\hline & 210 & $17.81^{\mathrm{a}, \mathrm{b}}$ & $17.11^{\mathrm{a}}$ & $19.75^{a}$ & $19.87^{\mathrm{a}}$ & $15.43^{\mathrm{a}}$ & $18.98^{\mathrm{a}}$ & $20.62^{a}$ & $17.76^{\mathrm{a}}$ & $21.48^{a}$ & $21.73^{\mathrm{a}}$ & $17.21^{\mathrm{a}}$ & $16.76^{\mathrm{a}}$ & $19.38^{\mathrm{a}}$ & $18.73^{\mathrm{a}}$ & 0.072 & 0.42 \\
\hline & $\begin{array}{c}p- \\
\text { value }\end{array}$ & 0.000 & 0.000 & 0.003 & 0.002 & 0.000 & 0.000 & 0.013 & 0.003 & 0.002 & 0.000 & 0.000 & 0.000 & 0.001 & 0.000 & & \\
\hline & SEM & 1.55 & 1.84 & 1.51 & 1.60 & 1.96 & 1.66 & 1.72 & 1.65 & 1.49 & 1.77 & 1.77 & 1.76 & 1.51 & 1.73 & & \\
\hline
\end{tabular}

${ }^{a-e}$ Mean values in the same column (same antioxidant in different weeks) with different letter presented significant differences; ${ }^{1-8}$ Mean values in the same row (different antioxidant in the same week) with different number presented significant differences; SEM: standard error of mean; Batches: CON: control; BHT: tert-butyl-4-hydroxytoluene; TEA: tea; CHE: chestnut; GRA: grape seed and BER: beer extracts. 
The values of lightness $\left(\mathrm{L}^{*}\right)$ decreased significantly $(p<0.01)$ until the end of the storage. This behavior could be due as a consequence of moisture losses [17]. Therefore, a significant correlation was found with moisture $(r=0.87, p<0.01)$ and $\mathrm{a}_{\mathrm{w}}(r=0.80, p<0.01)$. These results were lower than those found by other authors [6,24]. During ripening time the values ranged from 43.8 to 30.5, being CON the samples that showed the lowest values. As in previous studies conducted with these extracts [5], the samples that contained extracts in their composition showed higher $\mathrm{L}^{*}$ values. At the end of the ripening process, the samples that contain TEA50, TEA200, GRA50 and GRA200 in their formulation were those showed the highest values, even improving the results obtaining for BHT $(38.98,36.54$, 36.96, and 37.36 vs. 35.34, respectively). The same behavior was observed during vacuum packaging, the decline in the values continued, reaching values between 34.9 and 26.8, and the values were higher in samples with added extracts, being $\mathrm{CHE}_{200}$ the samples that showed the highest values. Regarding dose effect, significant differences $(p<0.05)$ were found for this color parameter in samples that contained GRA in their composition.

\subsection{Effect of Antioxidants on TPA Analysis during the Manufacturing Process and Vacuum Packaging}

The evolutions of TPA parameters (hardness, springiness, chewiness, gumminess and cohesiveness) during ripening and vacuum-packaged are shown in Table 2. The major changes take place during fermentation when the $\mathrm{pH}$ declines and the myofibrillar proteins aggregate leading to gel formation. Thereby, a significant increase $(p<0.05)$ in hardness, chewiness and gumminess was observed during the first 48 days of ripening, to remain stable until the end of storage. In the case of springiness, the values decreased during ripening to remain constant during vacuum-packaged. Drying is a major factor affecting binding and rheological properties [26]. In fact, a significant correlation $(p<0.01)$ were found between moisture and water activity and TPA parameters. In the case of hardness, chewiness, gumminess and cohesiveness these correlations with moisture $(r=-0.67, r=-0.48, r=-0.75$, $r=-0.26$, respectively) and water activity were negative $(r=-0.59, r=-0.38, r=-0.65, r=-0.31$, respectively), so an increase in these parameters during ripening time was due to a decrease in moisture and water activity. In addition, other research have shown that polyphenolic compounds are able to react with thiol groups in meat protein to form covalent thiol-quinone adducts [27]. Specifically, it has been hypothesized that polyphenolic compounds from green tea extract can alter the textural properties of Bologna type sausages [28], so especially at elevated concentrations phenolics compounds could interact with the protein thiols to modify water holding capacity and other textural parameters.

The addition of antioxidants significantly $(p<0.001)$ decreased hardness values. The lower results were found in the samples treated with $\mathrm{TEA}_{200}, \mathrm{CHE}_{200}$ and GRA50, followed by the samples treated with BHT and BER extracts. The values obtained for CON samples at the end of ripening were similar to those obtained in other studies [6] and lower to the results reported by González-Fernández et al. [26] in Galician chorizos. On the other hand, significant differences $(p<0.05)$ were found in TEA, CHE and GRA depending on the dose. The lower results of hardness were obtained with the dose of $200 \mathrm{mg} / \mathrm{kg}$ both during ripening and vacuum-packaging. 
Table 2. Evolution of textural properties of "chorizo" treated with BHT and natural antioxidants during ripening and vacuum-packaging.

\begin{tabular}{|c|c|c|c|c|c|c|c|c|c|c|c|c|c|c|c|c|c|}
\hline & \multirow{2}{*}{ Days } & \multirow{2}{*}{$\mathrm{CON}$} & \multirow{2}{*}{$\begin{array}{c}\text { BHT } \\
200 \\
\end{array}$} & \multicolumn{3}{|c|}{ TEA } & \multicolumn{3}{|c|}{ CHE } & \multicolumn{3}{|c|}{ GRA } & \multicolumn{3}{|c|}{ BER } & \multirow{2}{*}{$p$-Value } & \multirow{2}{*}{ SEM } \\
\hline & & & & 50 & 200 & 1000 & 50 & 200 & 1000 & 50 & 200 & 1000 & 50 & 200 & 1000 & & \\
\hline & 4 & $2.59^{\mathrm{a}, 2-4}$ & $2.10^{\mathrm{a}, 1-3}$ & $2.47^{\mathrm{a}, 2,3}$ & $1.49^{\mathrm{a}, 1}$ & $2.01^{\mathrm{a}, 1-3}$ & $1.78^{\mathrm{a}, 1,2}$ & $2.10^{\mathrm{a}, 1-3}$ & $1.96^{\mathrm{a}, 1-3}$ & $2.70^{a, 3-5}$ & $2.27^{\mathrm{a}, 1-3}$ & $3.98^{\mathrm{a}, 6}$ & $3.36^{\mathrm{a}, 4-6}$ & $3.46^{\mathrm{a}, 56}$ & $3.69^{\mathrm{a}, 6}$ & 0.000 & 0.15 \\
\hline & 19 & $8.66^{\mathrm{b}, 7}$ & $7.77^{b, 6,7}$ & $4.27^{b, 1-4}$ & $5.61^{b, 3-6}$ & $5.35^{\mathrm{b}, 2-5}$ & $3.97^{\mathrm{b}, 1-3}$ & $2.76^{\mathrm{a}, 1}$ & $2.49^{\mathrm{a}, 1}$ & $3.12^{\mathrm{a}, 1,2}$ & $3.51^{\mathrm{a}, 1-3}$ & $8.73^{\mathrm{b}, 7}$ & $6.29^{\mathrm{a}, 4-6}$ & $5.08^{a, 2-5}$ & $7.20^{b, 5-7}$ & 0.000 & 0.42 \\
\hline & 48 & $17.50^{\mathrm{d}, 7}$ & $9.84^{b, 3-5}$ & $6.89^{\mathrm{c}, 1}$ & $6.56^{\mathrm{b}, 1}$ & $8.60^{\mathrm{c}, 2,3}$ & $7.01^{\mathrm{c}, 1}$ & $6.97^{\mathrm{b}, 1}$ & $7.59^{\mathrm{b}, 1,2}$ & $7.47^{b, 1,2}$ & $8.91^{b, c, 2-4}$ & $10.30^{\mathrm{c}, 4,5}$ & $10.30^{\mathrm{b}, 4,5}$ & $12.00^{\mathrm{b}, \mathrm{c}, 6}$ & $10.52^{b, c, 5}$ & 0.000 & 0.55 \\
\hline Hardness & 60 & $15.77^{\mathrm{c}, 5}$ & $9.98^{b, 3}$ & $7.78^{\mathrm{c}, 1,2}$ & $6.82^{\mathrm{b}, 1}$ & $8.52^{c, 2}$ & $7.37^{\mathrm{c}, 1,2}$ & $7.60^{b, 1,2}$ & $7.68^{b, 1,2}$ & $7.78^{b, 1,2}$ & $7.89^{\mathrm{b}, 1,2}$ & $11.17^{\mathrm{c}, 3}$ & $10.91^{\mathrm{b}, 3}$ & $13.93^{\mathrm{c}, 4}$ & $10.24^{b, 3}$ & 0.000 & 0.50 \\
\hline \multirow[t]{7}{*}{$(\mathrm{kg})$} & 120 & $16.07^{\mathrm{c}, \mathrm{d}, 7}$ & $9.36^{b, 3-6}$ & $10.70^{\mathrm{d}, 5,6}$ & $6.30^{\mathrm{b}, 1}$ & $8.74^{\mathrm{c}, 2-5}$ & $7.45^{\mathrm{c}, 1-3}$ & $8.21^{b, 1-4}$ & $7.30^{\mathrm{b}, 1-3}$ & $7.12^{b, 1,2}$ & $9.81^{c, 4-6}$ & $11.23^{\mathrm{c}, 6}$ & $11.29^{\mathrm{b}, 6}$ & $10.22^{b, 46}$ & $9.78^{b, 4-6}$ & 0.000 & 0.47 \\
\hline & 210 & $16.07^{\mathrm{c}, \mathrm{d}, \mathrm{S}}$ & $9.01^{\mathrm{b}, 12}$ & $11.27^{\mathrm{d}, 2,3}$ & $8.58^{\mathrm{c}, 1,2}$ & $9.57^{\mathrm{c}, 1,2}$ & $9.22^{\mathrm{d}, 1,2}$ & $7.78^{b, 1}$ & $8.24^{\mathrm{b}, 1}$ & $9.55^{\mathrm{c}, 1,2}$ & $9.66^{\mathrm{c}, 1,2}$ & $12.83^{\mathrm{d}, 3,4}$ & $14.44^{\mathrm{c}, 4,5}$ & $13.64^{c, 3-5}$ & $13.84^{\mathrm{c}, 3-5}$ & 0.000 & 0.52 \\
\hline & $p$-value & 0.000 & 0.001 & 0.000 & 0.000 & 0.005 & 0.000 & 0.000 & 0.000 & 0.000 & 0.000 & 0.000 & 0.001 & 0.000 & 0.005 & & \\
\hline & SEM & 1.73 & 0.85 & 0.96 & 0.67 & 0.84 & 0.75 & 0.77 & 0.79 & 0.76 & 0.91 & 0.86 & 1.12 & 1.24 & 0.99 & & \\
\hline & 4 & $0.42^{\mathrm{b}, 4}$ & $0.38^{b, 3,4}$ & $0.34^{1-3}$ & $0.36^{2,3}$ & $0.29^{1}$ & $0.33^{1,2}$ & $0.36^{\mathrm{d}, 2,3}$ & $0.31^{1,2}$ & $0.31^{1,2}$ & $0.30^{1,2}$ & $0.30^{1}$ & $0.30^{1,2}$ & $0.31^{1,2}$ & $0.29^{1}$ & 0.001 & 0.01 \\
\hline & 19 & $0.31^{\mathrm{a}}$ & $0.35^{\mathrm{b}}$ & 0.28 & 0.31 & 0.30 & 0.33 & $0.32^{b, c}$ & 0.33 & 0.32 & 0.31 & 0.35 & 0.32 & 0.32 & 0.36 & 0.390 & 0.01 \\
\hline & 48 & $0.31^{\mathrm{a}}$ & $0.28^{\mathrm{a}}$ & 0.31 & 0.29 & 0.27 & 0.28 & $0.29^{\mathrm{a}, \mathrm{b}}$ & 0.30 & 0.29 & 0.34 & 0.30 & 0.35 & 0.33 & 0.32 & 0.246 & 0.01 \\
\hline \multirow{8}{*}{$\begin{array}{c}\text { Springiness } \\
(\mathrm{mm})\end{array}$} & 60 & $0.30^{\mathrm{a}, 1-4}$ & $0.27^{\mathrm{a}, 1,2}$ & $0.32^{2-5}$ & $0.31^{2-5}$ & $0.26^{1}$ & $0.28^{1-3}$ & $0.28^{\mathrm{a}, 1-3}$ & $0.34^{4,5}$ & $0.30^{1-4}$ & $0.31^{2-5}$ & $0.29^{1234}$ & $0.31^{2-5}$ & $0.36^{5}$ & $0.33^{3-5}$ & 0.016 & 0.01 \\
\hline & 120 & $0.31^{\mathrm{a}}$ & $0.29^{\mathrm{a}}$ & 0.31 & 0.30 & 0.30 & 0.27 & $0.31^{\mathrm{b}, \mathrm{c}}$ & 0.30 & 0.30 & 0.30 & 0.30 & 0.29 & 0.29 & 0.28 & 0.666 & 0.01 \\
\hline & 210 & $0.31^{\mathrm{a}, 1-3}$ & $0.29^{\mathrm{a}, 1}$ & $0.29^{1}$ & $0.29^{1}$ & $0.30^{1,2}$ & $0.32^{1-4}$ & $0.33^{\mathrm{c}, \mathrm{d}, 1-4}$ & $0.31^{1-3}$ & $0.32^{1-3}$ & $0.31^{1-3}$ & $0.33^{1-4}$ & $0.35^{2-4}$ & $0.37^{4}$ & $0.36^{3,4}$ & 0.035 & 0.01 \\
\hline & $p$-value & 0.000 & 0.001 & 0.074 & 0.318 & 0.190 & 0.376 & 0.007 & 0.410 & 0.625 & 0.649 & 0.218 & 0.424 & 0.130 & 0.069 & & \\
\hline & SEM & 0.01 & 0.01 & 0.01 & 0.01 & 0.01 & 0.01 & 0.01 & 0.01 & 0.01 & 0.01 & 0.01 & 0.01 & 0.01 & 0.01 & & \\
\hline & 4 & $0.25^{\mathrm{a}, 3}$ & $0.14^{\mathrm{a}, 1,2}$ & $0.14^{\mathrm{a}, 1,2}$ & $0.18^{\mathrm{a}, 1}$ & $0.10^{\mathrm{a}, 1}$ & $0.10^{\mathrm{a}, 1}$ & $0.15^{\mathrm{a}, 1,2}$ & $0.09^{\mathrm{a}, 1}$ & $0.12^{\mathrm{a}, 1,2}$ & $0.09^{\mathrm{a}, 1}$ & $0.20^{\mathrm{a}, 2,3}$ & $0.16^{\mathrm{a}, 1,2}$ & $0.19^{\mathrm{a}, 2,3}$ & $0.17^{\mathrm{a}, 1-3}$ & 0.009 & 0.01 \\
\hline & 19 & $0.97^{b, 4}$ & $0.62^{b, 3}$ & $0.19^{\mathrm{a}, 1}$ & $0.17^{\mathrm{a}, \mathrm{a}, \mathrm{l}, 1,2}$ & $0.31^{\mathrm{a}, \mathrm{b}, \mathrm{l}, \mathrm{2}}$ & $0.22^{\mathrm{a}, 1,2}$ & $0.21^{\mathrm{a}, 1,2}$ & $0.18^{\mathrm{a}, 1}$ & $0.20^{\mathrm{a}, 1,2}$ & $0.24^{\mathrm{a}, 1,2}$ & $0.86^{\mathrm{b}, 4}$ & $0.43^{\mathrm{b}, 2,3}$ & $0.33^{\mathrm{a}, \mathrm{b}, \mathrm{l}, \mathrm{1}, 2}$ & $0.63^{\mathrm{b}, 3}$ & 0.000 & 0.05 \\
\hline & 48 & $1.29^{\mathrm{c}, 6}$ & $0.74^{\mathrm{b}, 2,3}$ & $0.65^{\mathrm{b}, 1,2}$ & $0.27^{\mathrm{b}, \mathrm{c}, 1}$ & $0.64^{b, c, 1,1,2}$ & $0.53^{\mathrm{b}, 1}$ & $0.55^{\mathrm{b}, 1}$ & $0.66^{\mathrm{b}, 1,2}$ & $0.63^{\mathrm{b}, 1,2}$ & $0.97^{\mathrm{b}, 4,5}$ & $0.88^{b, 3,4}$ & $1.08^{\mathrm{d}, 5}$ & $1.04^{\mathrm{c}, \mathrm{d}, \mathrm{d}, 5}$ & $0.89^{b, 3-5}$ & 0.000 & 0.05 \\
\hline \multirow{5}{*}{$\begin{array}{l}\text { Chewiness } \\
(\mathrm{kg} \times \mathrm{mm})\end{array}$} & 60 & $1.11^{\mathrm{b}, \mathrm{c}, 5}$ & $0.65^{\mathrm{b}, 1,2}$ & $0.75^{\mathrm{b}, \mathrm{c}, 1-3}$ & $0.30^{\mathrm{c}, 12}$ & $0.56^{\mathrm{acc}, 1}$ & $0.61^{\mathrm{b}, 1,2}$ & $0.58^{\mathrm{b}, \mathrm{c}, 1}$ & $0.85^{\mathrm{c}, 2-4}$ & $0.69^{\mathrm{b}, 1,2}$ & $0.79^{\mathrm{b}, 1-4}$ & $0.85^{b, 2-4}$ & $1.02^{\mathrm{d}, 4,5}$ & $1.41^{\mathrm{d}, \mathrm{e}, \mathrm{G}}$ & $0.97^{\mathrm{b}, 3-5}$ & 0.000 & 0.05 \\
\hline & 120 & $1.29^{\mathrm{c}, 4}$ & $0.75^{b, 1-3}$ & $1.01^{\mathrm{d}, 3}$ & $0.32^{\mathrm{c}, 1,2}$ & $0.80^{\mathrm{c}, 1-3}$ & $0.51^{\mathrm{b}, 1}$ & $0.76^{\mathrm{b}, \mathrm{c}, 1-3}$ & $0.62^{b, 1,2}$ & $0.66^{\mathrm{b}, 1,2}$ & $0.83^{b, 2,3}$ & $0.86^{\mathrm{b}, 2,3}$ & $0.83^{\mathrm{c}, 2,3}$ & $0.81^{b, c, 1-3}$ & $0.77^{\mathrm{b}, 1-3}$ & 0.003 & 0.04 \\
\hline & 210 & $1.16^{\mathrm{b}, \mathrm{c}, 1-3}$ & $0.72^{\mathrm{b}, 1}$ & $0.84^{\mathrm{c}, 1,2}$ & $0.27^{\mathrm{c}, 1}$ & $0.84^{\mathrm{c}, 1,2}$ & $0.87^{\mathrm{c}, 1,2}$ & $0.82^{\mathrm{c}, 1,2}$ & $0.73^{\mathrm{b}, 1}$ & $0.98^{\mathrm{c}, 1-3}$ & $0.87^{\mathrm{b}, 1,2}$ & $1.33^{\mathrm{c}, 2-4}$ & $1.48^{\mathrm{e}, 3,4}$ & $1.69^{\mathrm{e}, 4}$ & $1.47^{c, 3,4}$ & 0.005 & 0.07 \\
\hline & p-value & 0.000 & 0.004 & 0.000 & 0.018 & 0.037 & 0.001 & 0.002 & 0.000 & 0.002 & 0.006 & 0.000 & 0.000 & 0.004 & 0.001 & & \\
\hline & SEM & 0.13 & 0.07 & 0.10 & 0.02 & 0.09 & 0.08 & 0.08 & 0.09 & 0.09 & 0.11 & 0.10 & 0.13 & 0.17 & 0.12 & & \\
\hline
\end{tabular}


Table 2. Cont.

\begin{tabular}{|c|c|c|c|c|c|c|c|c|c|c|c|c|c|c|c|c|c|}
\hline & \multirow{2}{*}{ Days } & \multirow{2}{*}{ CON } & \multirow{2}{*}{$\begin{array}{l}\text { BHT } \\
200 \\
\end{array}$} & \multicolumn{3}{|c|}{ TEA } & \multicolumn{3}{|c|}{ CHE } & \multicolumn{3}{|c|}{ GRA } & \multicolumn{3}{|c|}{ BER } & \multirow{2}{*}{$p$-value } & \multirow{2}{*}{ SEM } \\
\hline & & & & 50 & 200 & 1000 & 50 & 200 & 1000 & 50 & 200 & 1000 & 50 & 200 & 1000 & & \\
\hline \multirow{8}{*}{$\begin{array}{c}\text { Gumminess } \\
(\mathrm{kg})\end{array}$} & 4 & $0.58^{a, 3-5}$ & $0.35^{\mathrm{a}, \mathrm{l}-3}$ & $0.39^{\mathrm{a}, 1-4}$ & $0.27^{\mathrm{a}, 1}$ & $0.35^{\mathrm{a}, 1-3}$ & $0.30^{\mathrm{a}, 1,2}$ & $0.43^{\mathrm{a}, 1-5}$ & $0.28^{\mathrm{a}, 1}$ & $0.41^{\mathrm{a}, 1-4}$ & $0.30^{\mathrm{a}, 1,2}$ & $0.67^{\mathrm{a}, 5}$ & $0.54^{\mathrm{a}, 2-5}$ & $0.61^{\mathrm{a}, 4,5}$ & $0.59^{\mathrm{a}, 3-5}$ & 0.013 & 0.03 \\
\hline & 19 & $3.00^{\mathrm{b}, 5}$ & $1.80^{\mathrm{b}, 3,4}$ & $0.66^{\mathrm{a}, 1,2}$ & $0.77^{\mathrm{a}, 1,2}$ & $1.04^{\mathrm{a}, \mathrm{b}, \mathrm{l}, \mathrm{2}}$ & $0.69^{\mathrm{a}, 1,2}$ & $0.68^{\mathrm{a}, 1,2}$ & $0.54^{\mathrm{a}, 1}$ & $0.58^{\mathrm{a}, 1}$ & $0.78^{\mathrm{a}, 1,2}$ & $2.41^{\mathrm{b}, 4,5}$ & $1.33^{\mathrm{a}, 2,3}$ & $1.05^{\mathrm{a}, 1,2}$ & $1.85^{b, 3,4}$ & 0.000 & 0.15 \\
\hline & 48 & $4.26^{c, 6}$ & $2.62^{c, 3-5}$ & $2.07^{\mathrm{b}, 1-3}$ & $1.68^{\mathrm{b}, 1}$ & $2.34^{\mathrm{c}, 2-4}$ & $1.87^{\mathrm{b}, 1,2}$ & $1.89^{\mathrm{b}, 1,2}$ & $2.19^{\mathrm{b}, \mathrm{c}, 1-3}$ & $2.16^{\mathrm{b}, 1-3}$ & $2.86^{\mathrm{b}, 4,5}$ & $2.93^{c, 5}$ & $3.14^{\mathrm{b}, 5}$ & $3.15^{\mathrm{b}, \mathrm{c}, 5}$ & $2.84^{\mathrm{b}, \mathrm{c}, 4,5}$ & 0.000 & 0.13 \\
\hline & 60 & $3.79^{c, 5}$ & $2.44^{\mathrm{c}, 1,2}$ & $2.35^{b, 1}$ & $2.05^{\mathrm{b}, 1}$ & $2.21^{\mathrm{b}, \mathrm{c}, 1}$ & $2.12^{\mathrm{b}, 1}$ & $2.11^{\mathrm{b}, 1}$ & $2.52^{\mathrm{d}, 1-3}$ & $2.30^{\mathrm{b}, 1}$ & $2.48^{\mathrm{b}, 1,2}$ & $2.93^{\mathrm{c}, 2-4}$ & $3.26^{\mathrm{b}, 4}$ & $3.93^{\mathrm{c}, \mathrm{d}, 5}$ & $3.01^{\mathrm{c}, 3,4}$ & 0.000 & 0.12 \\
\hline & 120 & $4.23^{\mathrm{c}, 6}$ & $2.60^{c, 1-5}$ & $3.30^{\mathrm{d}, 5}$ & $2.01^{\mathrm{b}, 1,2}$ & $2.69^{\mathrm{c}, 2-5}$ & $1.93^{\mathrm{b}, 1}$ & $2.46^{\mathrm{b}, 1-4}$ & $2.08^{\mathrm{b}, 1,2}$ & $2.20^{\mathrm{b}, 1-3}$ & $2.83^{\mathrm{b}, 3-5}$ & $2.94^{\mathrm{c}, 4,5}$ & $2.95^{\mathrm{b}, 4,5}$ & $2.82^{b, 3-5}$ & $2.81^{b, c, 3-5}$ & 0.000 & 0.12 \\
\hline & 210 & $3.76^{\mathrm{c}, 3-5}$ & $2.47^{\mathrm{c}, 1}$ & $2.92^{\mathrm{c}, 1-3}$ & $2.25^{\mathrm{b}, 1}$ & $2.70^{c, 1,2}$ & $2.66^{\mathrm{c}, 1,2}$ & $2.48^{b, 1}$ & $2.36^{\mathrm{c}, \mathrm{d}, 1}$ & $3.09^{\mathrm{c}, 1-4}$ & $2.74^{b, 1,2}$ & $3.98^{\mathrm{d}, 3-5}$ & $4.28^{c, 5}$ & $4.52^{\mathrm{d}, 5}$ & $4.14^{\mathrm{d}, 4,5}$ & 0.001 & 0.16 \\
\hline & $p$-value & 0.000 & 0.000 & 0.000 & 0.002 & 0.012 & 0.000 & 0.001 & 0.000 & 0.000 & 0.000 & 0.000 & 0.000 & 0.000 & 0.002 & & \\
\hline & SEM & 0.44 & 0.25 & 0.33 & 0.23 & 0.29 & 0.25 & 0.25 & 0.27 & 0.30 & 0.32 & 0.30 & 0.39 & 0.44 & 0.34 & & \\
\hline \multirow{8}{*}{ Cohesiveness } & 4 & $0.23^{\mathrm{a}, \mathrm{b}}$ & $0.17^{\mathrm{a}}$ & $0.16^{\mathrm{a}}$ & $0.10^{\mathrm{a}}$ & $0.18^{\mathrm{a}}$ & $0.17^{\mathrm{a}}$ & $0.21^{\mathrm{a}}$ & $0.16^{\mathrm{a}}$ & $0.15^{\mathrm{a}}$ & $0.14^{\mathrm{a}}$ & $0.17^{\mathrm{a}}$ & $0.16^{\mathrm{a}}$ & $0.17^{\mathrm{a}}$ & $0.16^{\mathrm{a}}$ & 0.256 & 0.01 \\
\hline & 19 & $0.34^{c, 5}$ & $0.23^{b, 2-4}$ & $0.18^{\mathrm{a}, 1,2}$ & $0.23^{a, 1}$ & $0.20^{\mathrm{a}, 1,2}$ & $0.18^{\mathrm{a}, 1,2}$ & $0.22^{\mathrm{a}, 1-}$ & $0.22^{b, 1-4}$ & $0.19^{\mathrm{a}, 1,2}$ & $0.20^{a, 1,2}$ & $0.27^{b, 4}$ & $0.22^{b, 1-4}$ & $0.21^{\mathrm{a}, 1-3}$ & $0.26^{b, 3,4}$ & 0.000 & 0.01 \\
\hline & 48 & $0.24^{b}$ & $0.27^{\mathrm{b}}$ & $0.30^{\mathrm{c}}$ & $0.48^{\mathrm{b}}$ & $0.28^{b}$ & $0.27^{\mathrm{b}}$ & $0.28^{\mathrm{a}, \mathrm{b}}$ & $0.30^{\mathrm{c}}$ & $0.29^{b}$ & $0.32^{b}$ & $0.29^{b}$ & $0.30^{\mathrm{c}}$ & $0.26^{\mathrm{b}}$ & $0.27^{\mathrm{b}}$ & 0.260 & 0.01 \\
\hline & 60 & $0.24^{\mathrm{b}, 1}$ & $0.25^{\mathrm{b}, 1}$ & $0.30^{\mathrm{c}, 3-5}$ & $0.64^{\mathrm{b}, 3-5}$ & $0.26^{\mathrm{b}, 1,2}$ & $0.29^{b, 2-4}$ & $0.28^{a, b, 1-3}$ & $0.33^{\mathrm{d}, 5}$ & $0.30^{\mathrm{b}, 2-5}$ & $0.32^{b, 4,5}$ & $0.26^{\mathrm{b}, 1,2}$ & $0.30^{c, 3-5}$ & $0.29^{b, 2-4}$ & $0.30^{b, 2-5}$ & 0.001 & 0.01 \\
\hline & 120 & $0.27^{\mathrm{b}}$ & $0.28^{b}$ & $0.31^{\mathrm{c}}$ & $0.61^{\mathrm{b}}$ & $0.31^{\mathrm{b}}$ & $0.26^{\mathrm{b}}$ & $0.30^{\mathrm{b}}$ & $0.29^{\mathrm{c}}$ & $0.31^{\mathrm{b}}$ & $0.29^{\mathrm{b}}$ & $0.26^{\mathrm{b}}$ & $0.27^{\mathrm{c}}$ & $0.28^{\mathrm{b}}$ & $0.29^{b}$ & 0.102 & 0.01 \\
\hline & 210 & $0.23^{\mathrm{a}, \mathrm{b}, 1}$ & $0.28^{b, 1-4}$ & $0.26^{\mathrm{b}, 1,2}$ & $0.66^{\mathrm{b}, 1-3}$ & $0.28^{b, 1-4}$ & $0.29^{b, 2-5}$ & $0.32^{b, 4,5}$ & $0.29^{\mathrm{c}, 2-5}$ & $0.33^{b, 5}$ & $0.29^{b, 2-5}$ & $0.31^{b, 3-5}$ & $0.30^{c, 2-5}$ & $0.33^{c, 5}$ & $0.30^{b, 2-5}$ & 0.006 & 0.01 \\
\hline & $p$-value & 0.002 & 0.027 & 0.000 & 0.003 & 0.003 & 0.002 & 0.030 & 0.000 & 0.000 & 0.006 & 0.010 & 0.001 & 0.001 & 0.002 & & \\
\hline & SEM & 0.01 & 0.01 & 0.02 & 0.07 & 0.02 & 0.02 & 0.01 & 0.02 & 0.02 & 0.02 & 0.02 & 0.02 & 0.02 & 0.02 & & \\
\hline
\end{tabular}

${ }^{\text {a-e }}$ Mean values in the same column (same antioxidant in different weeks) with different letter presented significant differences; ${ }^{1-7}$ Mean values in the same row (different antioxidant in the same week) with different number presented significant differences. 
Gumminess and chewiness were significantly $(p<0.05)$ increased during ripening time, reaching mean values of $4.26 \mathrm{~kg}$ and $1.29 \mathrm{~kg} \times \mathrm{mm}$, respectively. This increase indicated that gumminess changed from short to pasty gummy through ripening. The highest values were observed in CON samples, while the lowest were found in the samples treated with TEA and GRA extracts. Within the dose effect, significant differences $(p<0.05)$ were found among samples treated with different concentrations of TEA, CHE and GRA extracts. In the case of TEA, the samples treated with $200 \mathrm{mg} / \mathrm{kg}$ were that showed the lowest values, while a dose of $50 \mathrm{mg} / \mathrm{kg}$ were enough in CHE and GRA samples. Chewiness values indicated that sausages became tougher during ripening period. As occurred with gumminess, the lowest values were found in the samples treated with TEA extract. Significant differences $(p<0.05)$ were found among batches during ripening time. However, regarding dose effect, only significant differences were found in samples treated with TEA extract ( 0.65 vs. 0.27 vs. $0.64 \mathrm{~kg} \times \mathrm{mm}_{\text {in }} \mathrm{TEA}_{50}, \mathrm{TEA}_{200}$ and TEA 1000 , respectively).

Springiness values have been related to the elastic properties of sausages [29]. The values decreased during ripening to remain constant during vacuum-packaged, but only in CON and samples treated with BHT and $\mathrm{CHE}_{200}$ this decreased was significant $(p<0.01)$. This result could be also due to water removal during the ripening period. No significant differences $(p>0.05)$ were observed among treatments and the end of ripening, but these differences were significant $(p<0.05)$ at the end of the vacuum-packaging (Table 2).

\subsection{Effect of Antioxidants on Oxidative Stability during the Manufacturing Process and Vacuum Packaging}

The influence of antioxidants on oxidative stability during the manufacturing process and vacuum packaging was evaluated as TBARS index (Figure 2). Significant changes $(p<0.05)$ were detected in TBARS values among samples during storage time. According to other authors $[5,10]$, CON batches showed more intense lipid oxidation. Thereby, samples with contained antioxidants in their composition showed values below $0.4 \mathrm{mg} \mathrm{MDA} / \mathrm{kg}$, showing that these extracts reduced lipid oxidation of the dry-cured sausage. The maximum TBARS values were observed at the end of ripening time (values between 0.23 to $0.78 \mathrm{mg} \mathrm{MDA} / \mathrm{kg}$ at 0 and 48 days, respectively) followed by a decline until day 60, to remain constant to the end of vacuum packaging. The values found during ripening and vacuum packaging were higher than those found by other authors [6,30] and similar to those found in other dry-cured sausages [17]. The levels obtained during storage period were significantly $(p<0.05)$ lower than the limit (2.0 $\mathrm{mg} \mathrm{MDA} / \mathrm{kg}$ ) which is accepted as deterioration level [31].

Regarding the effect of the addition of antioxidants, the results obtained were equal or even better to those found with BHT. Thereby, the samples treated with CHE and GRA reached mean values of $0.17 \mathrm{mg} \mathrm{MDA} / \mathrm{kg}$ at the end of vacuum packaging, while the samples that contained BHT showed values of $0.24 \mathrm{mg} \mathrm{MDA} / \mathrm{kg}$. These results are in agreement with previously published studies [4], which reported higher effectiveness of natural products compared to synthetic antioxidants and suggesting the possibility of using these extracts as replacers of commercial compounds. Within dose effect, no significant differences $(p>0.05)$ were found among samples on the same extract, so that the lower concentration of natural extract would be sufficient to improve the results obtained in CON samples. 

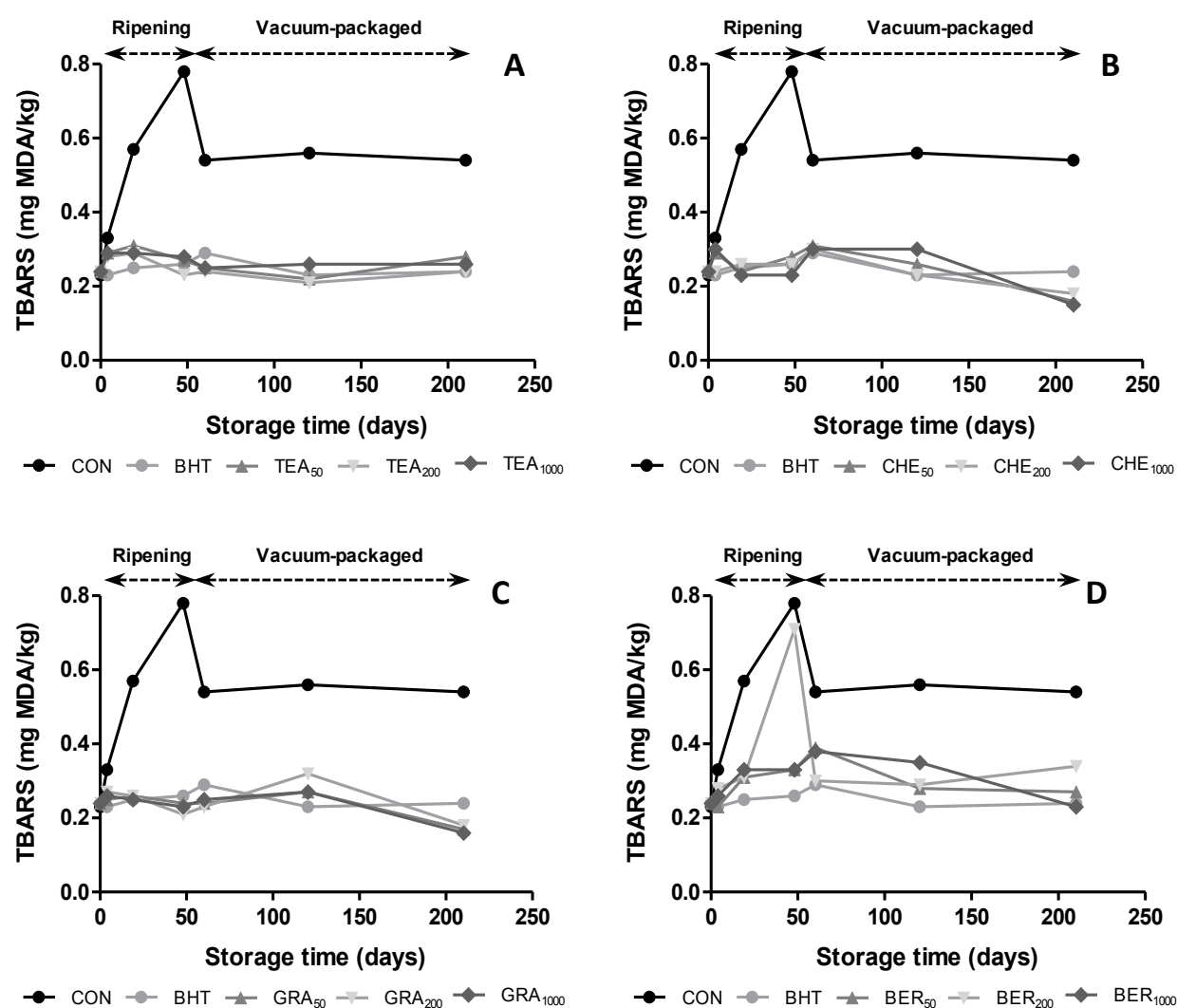

Figure 2. Evolution of thiobarbituric acid reactive substances (TBARS) in dry-cures sausages treated with BHT and natural antioxidants during ripening and vacuum-packaged storage.

\subsection{Effect of Antioxidants on Microbial Counts during the Manufacturing Process and}

\section{Vacuum Packaging}

Changes in the microbial populations, TVC, LAB and mold/yeasts, during the manufacturing process and vacuum packaging of dry-cured sausage "chorizo" are shown in Table 3 . The initial TVC, $\mathrm{LAB}$ and mold/yeasts counts ranged from $10^{3}$ to $10^{5} \mathrm{CFU} / \mathrm{g}$ (data not shown). Significant differences $(p<0.05)$ in microbial counts were detected among batches and during ripening and storage period. TVC counts increased from 5.17 to $8.62 \log _{10} \mathrm{CFU} / \mathrm{g}(p<0.001)$ during the first 19 days of ripening, remaining stable until the end of ripening (reaching values of $8.72 \log _{10} \mathrm{CFU} / \mathrm{g}$ ). TVC counts continued to increase up to day 60 , to proceed decreasing gradually during vacuum storage. Among batches, significant differences $(p<0.001)$ were found with the addition of antioxidants. At the end of ripening process, samples that contained extracts in their composition showed lower TVC counts than CON. Excluding $\mathrm{CHE}_{200}$, natural extracts showed lower results, getting to improve the results of BHT (8.12 $\log _{10} \mathrm{CFU} / \mathrm{g}$ vs. counts below $8 \log _{10} \mathrm{CFU} / \mathrm{g}$ in TEA $200, \mathrm{CHE}_{200}, \mathrm{GRA}_{1000}, \mathrm{BER}_{200}$ and BER 1000 ). Regarding dose effect, significant differences $(p<0.001)$ were found for all batches studied. Increase the level of natural antioxidant usually decreased the TVC counts. In fact, samples manufactured with GRA $_{1000}$ showed lower values than GRA50, GRA 200 and CON (7.91 $\log _{10}$ CFU/g vs. 8.14, 8.15 and $8.55 \log _{10} \mathrm{CFU} / \mathrm{g}$, respectively). In the other extracts, the dose of $200 \mathrm{mg} / \mathrm{kg}$ showed the lowest values (7.97, 7.88 and $7.95 \log _{10} \mathrm{CFU} / \mathrm{g}$ for TEA, CHE and BER, respectively). 
Table 3. Evolution of TVC, BAL and mold/yeast of "chorizo" treated with BHT and natural antioxidants during ripening and vacuum-packaging.

\begin{tabular}{|c|c|c|c|c|c|c|c|c|c|c|c|c|c|c|c|c|c|}
\hline & \multirow{2}{*}{ Days } & \multirow{2}{*}{$\mathrm{CON}$} & \multirow{2}{*}{$\begin{array}{c}\text { BHT } \\
200 \\
\end{array}$} & \multicolumn{3}{|c|}{ TEA } & \multicolumn{3}{|c|}{ CHE } & \multicolumn{3}{|c|}{ GRA } & \multicolumn{3}{|c|}{ BER } & \multirow{2}{*}{$p$-Value } & \multirow{2}{*}{ SEM } \\
\hline & & & & 50 & 200 & 1000 & 50 & 200 & 1000 & 50 & 200 & 1000 & 50 & 200 & 1000 & & \\
\hline \multirow{8}{*}{$T V C$} & 4 & $6.35^{a, 8}$ & $6.06^{\mathrm{a}, 4,5}$ & $6.34^{a, 8}$ & $5.88^{\mathrm{a}, 2}$ & $6.12^{a, 5,6}$ & $6.11^{a, 5,6}$ & $5.97^{\mathrm{a}, 3}$ & $5.94^{\mathrm{a}, 3}$ & $6.14^{\mathrm{a}, 6,7}$ & $6.09^{\mathrm{a}, 4-6}$ & $6.18^{a, 7}$ & $6.12^{a, 5,6}$ & $5.72^{a, 1}$ & $6.04^{\mathrm{a}, 4}$ & 0.000 & 0.03 \\
\hline & 19 & $8.62^{e, 8}$ & $8.38^{e, 4}$ & $8.42^{\mathrm{d}, 4,5}$ & $8.59^{\mathrm{c}, 7,8}$ & $8.51^{\mathrm{d}, 6}$ & $8.55^{\mathrm{f}, 6,7}$ & $8.46^{\mathrm{d}, 5}$ & $8.22^{\mathrm{d}, 2}$ & $8.19^{\mathrm{c}, 1,2}$ & $8.32^{e, 3}$ & $8.31^{\mathrm{e}, 3}$ & $8.21^{\mathrm{d}, 2}$ & $8.16^{\mathrm{c}, 1}$ & $8.58^{\mathrm{e}, 7,8}$ & 0.000 & 0.03 \\
\hline & 48 & $8.55^{\mathrm{d}, 8}$ & $8.12^{c, 5-7}$ & $8.06^{\mathrm{b}, 4}$ & $7.97^{b, 3}$ & $8.08^{c, 4,5}$ & $8.10^{\mathrm{d}, 4-6}$ & $7.88^{\mathrm{c}, 1}$ & $8.72^{\mathrm{f}, 9}$ & $8.14^{\mathrm{c}, 6,7}$ & $8.15^{\mathrm{d}, 7}$ & $7.91^{c, 1,2}$ & $8.10^{\mathrm{c}, 4-6}$ & $7.95^{b, 2,3}$ & $7.99^{\mathrm{c}, 3}$ & 0.000 & 0.04 \\
\hline & 60 & $9.07^{\mathrm{f}, 8}$ & $8.24^{\mathrm{d}, 2}$ & $8.30^{c, 2}$ & $8.70^{\mathrm{d}, 6}$ & $8.55^{\mathrm{d}, 4}$ & $8.28^{e, 2}$ & $8.84^{\mathrm{e}, 7}$ & $8.62^{e, 5}$ & $8.43^{\mathrm{d}, 3}$ & $8.65^{f, 5,6}$ & $8.27^{\mathrm{e}, 2}$ & $8.48^{\mathrm{e}, 3}$ & $8.46^{\mathrm{d}, 3}$ & $8.15^{\mathrm{d}, 1}$ & 0.000 & 0.05 \\
\hline & 120 & $7.61^{\mathrm{c}, 1}$ & $7.92^{b, 3}$ & $8.00^{b, 4}$ & $7.90^{b, 3}$ & $7.99^{\mathrm{c}, 4}$ & $7.99^{\mathrm{c}, 4}$ & $7.87^{c, 3}$ & $7.80^{b, 2}$ & $7.91^{b, 3}$ & $7.61^{b, 1}$ & $8.12^{\mathrm{d}, 6}$ & $8.06^{\mathrm{c}, 5}$ & $8.13^{c, 6}$ & $7.92^{b, 3}$ & 0.000 & 0.03 \\
\hline & 210 & $7.47^{b, 1}$ & $8.15^{c, 5}$ & $9.92^{e, 6}$ & $7.91^{b, 3,4}$ & $7.88^{b, 3,4}$ & $7.92^{b, 3,4}$ & $7.73^{b, 2}$ & $8.15^{c, 5}$ & $7.93^{b, 4}$ & $7.81^{c, 2,3}$ & $7.85^{b, 3,4}$ & $7.86^{b, 3,4}$ & $7.93^{b, 4}$ & $7.93^{b, 4}$ & 0.000 & 0.11 \\
\hline & $p$-value & 0.000 & 0.000 & 0.000 & 0.000 & 0.000 & 0.000 & 0.000 & 0.000 & 0.000 & 0.000 & 0.000 & 0.000 & 0.000 & 0.000 & & \\
\hline & SEM & 0.37 & 0.24 & 0.32 & 0.28 & 0.25 & 0.24 & 0.27 & 0.28 & 0.23 & 0.25 & 0.22 & 0.23 & 0.28 & 0.24 & & \\
\hline \multirow{8}{*}{$L A B$} & 4 & $6.62^{\mathrm{a}, 2}$ & $6.91^{\mathrm{a}, 6}$ & $6.87^{\mathrm{a}, 6}$ & $6.62^{\mathrm{a}, 2,3}$ & $7.07^{\mathrm{a}, 7}$ & $7.11^{\mathrm{a}, 7}$ & $6.54^{\mathrm{a}, 1}$ & $6.71^{\mathrm{a}, 4}$ & $6.78^{\mathrm{a}, 5}$ & $6.89^{\mathrm{a}, 6}$ & $6.88^{\mathrm{a}, 6}$ & $6.55^{\mathrm{a}, 1}$ & $6.67^{\mathrm{a}, 3,4}$ & $6.78^{\mathrm{a}, 5}$ & 0.000 & 0.03 \\
\hline & 19 & $7.97^{c, 6,7}$ & $7.90^{\mathrm{b}, \mathrm{c}, 3,34}$ & $7.95^{c, 5,6}$ & $7.93^{b, c, 4,5}$ & $8.00^{\mathrm{b}, 7-9}$ & $8.00^{\mathrm{b}, 7-9}$ & $7.95^{\mathrm{d}, 5,6}$ & $7.84^{b, 2}$ & $8.01^{b, c, 8,89}$ & $7.90^{c, 3,4}$ & $7.86^{\mathrm{a}, \mathrm{b}, 2,3}$ & $7.97^{b, 6-8}$ & $7.76^{\mathrm{b}, 1}$ & $8.03^{c, 9}$ & 0.000 & 0.04 \\
\hline & 48 & $8.12^{\mathrm{d}, 6,7}$ & $8.07^{\mathrm{d}, 5,6}$ & $8.01^{\mathrm{c}, 4,5}$ & $8.02^{\mathrm{c}, \mathrm{d}, 45}$ & $8.18^{\mathrm{c}, 7}$ & $7.97^{b, 3,4}$ & $7.80^{\mathrm{cd}, \mathrm{d}, 2}$ & $8.57^{\mathrm{c}, 8}$ & $7.93^{b, 3}$ & $7.96^{\mathrm{c}, 3,4}$ & $7.70^{\mathrm{a}, \mathrm{b}, 1}$ & $8.16^{\mathrm{b}, 7}$ & $8.03^{b, 4,5}$ & $7.84^{b, 2}$ & 0.000 & 0.04 \\
\hline & 60 & $11.08^{\mathrm{f}, 8}$ & $7.96^{\mathrm{c}, \mathrm{d}, 1,2}$ & $7.91^{\mathrm{c}, 1}$ & $8.06^{\mathrm{d}, 3}$ & $8.00^{b, 2,3}$ & $8.20^{b, 4,5}$ & $8.51^{e, 6}$ & $8.72^{\mathrm{c}, 7}$ & $8.23^{c, 5}$ & $8.49^{\mathrm{d}, 6}$ & $8.14^{\mathrm{b}, 4}$ & $8.05^{b, 3}$ & $8.44^{\mathrm{c}, 6}$ & $8.18^{\mathrm{d}, 4,5}$ & 0.000 & 0.15 \\
\hline & 120 & $8.32^{e, 2-4}$ & $8.32^{e, 2-4}$ & $7.95^{\mathrm{c}, 1-4}$ & $7.85^{b, 1-3}$ & $8.51^{\mathrm{d}, 3,4}$ & $8.50^{b, 3,4}$ & $7.34^{b, 1}$ & $8.00^{b, 1-4}$ & $9.71^{\mathrm{d}, 6}$ & $7.58^{\mathrm{b}, \mathrm{c}, 1,2}$ & $8.66^{b, 4,5}$ & $8.03^{b, 1-4}$ & $8.00^{b, 1-4}$ & $9.28^{e, 5,6}$ & 0.000 & 0.13 \\
\hline & 210 & $7.45^{\mathrm{b}}$ & $7.75^{b}$ & $7.54^{\mathrm{b}}$ & $7.90^{\mathrm{b}}$ & $8.22^{\mathrm{c}}$ & $7.87^{b}$ & $7.73^{c}$ & $7.94^{b}$ & $8.09^{b, c}$ & $7.32^{\mathrm{a}, \mathrm{b}}$ & $7.68^{\mathrm{a}, \mathrm{b}}$ & $7.69^{b}$ & $8.11^{b, c}$ & $7.95^{b, c}$ & 0.104 & 0.06 \\
\hline & $p$-value & 0.000 & 0.000 & 0.001 & 0.000 & 0.000 & 0.038 & 0.000 & 0.000 & 0.000 & 0.002 & 0.042 & 0.003 & 0.000 & 0.000 & & \\
\hline & SEM & 0.50 & 0.13 & 0.13 & 0.15 & 0.14 & 0.14 & 0.18 & 0.20 & 0.26 & 0.16 & 0.18 & 0.17 & 0.17 & 0.22 & & \\
\hline \multirow{8}{*}{ Mold/Yeast } & 4 & $6.09^{\mathrm{d}, 6}$ & $5.78^{a, 3}$ & $5.86^{\mathrm{d}, 3,4}$ & $5.67^{\mathrm{b}, 2}$ & $5.80^{\mathrm{b}, 3}$ & $6.00^{\mathrm{c}, 5}$ & $6.44^{\mathrm{c}, 7}$ & $5.56^{\mathrm{c}, 1}$ & $5.89^{\mathrm{d}, 4}$ & $5.89^{\mathrm{e}, 4}$ & $6.48^{\mathrm{e}, 7}$ & $5.78^{\mathrm{b}, 3}$ & $5.94^{\mathrm{c}, 4,5}$ & $6.13^{\mathrm{d}, 6}$ & 0.000 & 0.05 \\
\hline & 19 & $7.74^{e, 4}$ & $7.10^{b, 1,2}$ & $7.69^{\mathrm{e}, 3,4}$ & $7.52^{c, 2-4}$ & $7.16^{\mathrm{c}, 1,2}$ & $7.10^{\mathrm{d}, 1,2}$ & $7.60^{\mathrm{d}, 3,4}$ & $7.28^{\mathrm{d}, 1-3}$ & $7.31^{\mathrm{e}, 1-4}$ & $7.07^{\mathrm{f}, 1}$ & $7.38^{\mathrm{f}, 1-4}$ & $7.05^{\mathrm{c}, 1}$ & $7.75^{e, 4}$ & $7.67^{\mathrm{e}, 3,4}$ & 0.006 & 0.06 \\
\hline & 48 & $6.17^{\mathrm{d}, 6}$ & $5.61^{\mathrm{a}, 1}$ & $5.91^{\mathrm{d}, 4}$ & $5.71^{\mathrm{b}, 2}$ & $5.96^{\mathrm{b}, 4}$ & $5.81^{\mathrm{c}, 3}$ & $5.91^{\mathrm{b}, \mathrm{c}, 4}$ & $5.73^{\mathrm{c}, 2}$ & $5.79^{\mathrm{d}, 3}$ & $5.61^{\mathrm{c}, 1}$ & $6.19^{\mathrm{d}, 6}$ & $6.12^{b, 5}$ & $6.15^{\mathrm{d}, 5,6}$ & $6.24^{\mathrm{d}, 7}$ & 0.000 & 0.04 \\
\hline & 60 & $5.92^{\mathrm{c}, 9}$ & $5.58^{\mathrm{a}, 6}$ & $5.20^{\mathrm{c}, 3}$ & $5.78^{\text {b, } 7,8}$ & $5.51^{\mathrm{b}, 5}$ & $5.28^{\mathrm{a}, \mathrm{b}, \mathrm{k}}$ & $5.23^{\mathrm{b}, 3}$ & $5.31^{\mathrm{b}, 4}$ & $5.08^{\mathrm{b}, 2}$ & $4.93^{\mathrm{a}, 1}$ & $5.78^{\mathrm{c}, 7,8}$ & $5.89^{\mathrm{b}, 9}$ & $5.74^{\mathrm{b}, 7}$ & $5.82^{\mathrm{c}, 8}$ & 0.000 & 0.06 \\
\hline & 120 & $5.58^{\mathrm{b}, 5}$ & $5.21^{a, 3-5}$ & $4.53^{b, 1,2}$ & $5.05^{\mathrm{a}, \mathrm{b}, 2-5}$ & $5.08^{\mathrm{b}, 2-5}$ & $5.50^{\mathrm{b}, 4,5}$ & $4.37^{\mathrm{a}, 1}$ & $4.60^{\mathrm{a}, 1-3}$ & $4.89^{\mathrm{a}, 1-4}$ & $5.15^{\mathrm{b}, 2-5}$ & $5.43^{\mathrm{a}, 4,5}$ & $5.23^{a, 3-5}$ & $5.58^{\mathrm{b}, 5}$ & $5.53^{\mathrm{b}, 4,5}$ & 0.004 & 0.08 \\
\hline & 210 & $4.49^{\mathrm{a}, 2}$ & $5.12^{\mathrm{a}, 2,3}$ & $2.79^{\mathrm{a}, 1}$ & $4.66^{\mathrm{a}, 2,3}$ & $3.25^{\mathrm{a}, 1}$ & $5.04^{a, 2,3}$ & $4.48^{\mathrm{a}, 2}$ & $4.74^{\mathrm{a}, 2,3}$ & $5.32^{\mathrm{c}, 2,3}$ & $5.48^{\mathrm{c}, 2,3}$ & $5.57^{\mathrm{b}, 3}$ & $5.23^{\mathrm{a}, 2,3}$ & $4.67^{\mathrm{a}, 2,3}$ & $5.23^{\mathrm{a}, 2,3}$ & 0.000 & 0.16 \\
\hline & $p$-value & 0.000 & 0.043 & 0.000 & 0.003 & 0.000 & 0.000 & 0.000 & 0.000 & 0.000 & 0.000 & 0.000 & 0.001 & 0.000 & 0.000 & & \\
\hline & SEM & 0.36 & 0.22 & 0.45 & 0.28 & 0.36 & 0.20 & 0.35 & 0.27 & 0.24 & 0.21 & 0.20 & 0.19 & 0.28 & 0.24 & & \\
\hline
\end{tabular}

${ }^{a-f}$ Mean values in the same column (same antioxidant in different weeks) with different letter presented significant differences; ${ }^{1-9}$ Mean values in the same row (different antioxidant in the same week) with different number presented significant differences; SEM: standard error of mean; Batches: CON: control; BHT: tert-butyl-4-hydroxytoluene; TEA: tea; CHE: chestnut; GRA: grape seed and BER: beer extracts. 
The lactic acid bacteria (LAB) counts showed significant $(p<0.05)$ differences during ripening and storage. A rapid increase in the LAB population was observed during the first 19 days of fermentation, increasing counts from 4.7 to $8.0 \log _{10} \mathrm{CFU} / \mathrm{g}$. Until the end of fermentation and ripening process the counts remained constant, reaching values of between 8.57 and $7.70 \log _{10} \mathrm{CFU} / \mathrm{g}$. The samples than contained $\mathrm{CHE}_{1000}$ in their formulation showed the highest values, followed by CON, TEA 1000 and $\mathrm{BER}_{50}$, that presented similar values around $8.15 \log _{10} \mathrm{CFU} / \mathrm{g}$. Except for CON samples, the trend during vacuum packaging was to decreased slightly (33\% vs. mean values of $5.1 \%$ ).

Dose effect showed significant $(p<0.05)$ differences among batches during ripening process on LAB counts. GRA and BER extracts showed a decrease in the population of LAB with the increase of the concentration, obtaining lower values in GRA 1000 (7.70 vs. 7.93 and 7.96 for GRA 1000, GRA $_{50}$ and $\mathrm{GRA}_{200}$, respectively) and $\mathrm{BER}_{1000}\left(7.84\right.$ vs. 8.16 and 8.03 for $\mathrm{BER}_{1000}, \mathrm{BER}_{50}$ and $\mathrm{BER}_{200}$, respectively). In contrast, samples treated with TEA and CHE showed lower counts for the minor dose (Table 3). As happened with the aforementioned microbial groups, mold and yeasts counts increased rapidly during the first 19 days of ripening, from 3.4 to $7.7 \log _{10} \mathrm{CFU} / \mathrm{g}$, to decrease slightly until the end of ripening process with values ranged between 6.2 and $5.61 \log _{10} \mathrm{CFU} / \mathrm{g}$.

\subsection{Effect of Antioxidants on Free Fatty Acid Content during the Manufacturing Process and Vacuum Packaging}

The free fatty acid (FFA) content of the different batches expressed as $\mathrm{mg}$ of fatty acid/g of fat is shown in Tables 4 and 5. The predominated fatty acids both the end of ripening time and the end of storage at vacuum-packaging were monounsaturated fatty acids (MUFA), followed by saturated fatty acids (SFA) and polyunsaturated fatty acids (PUFA). These results are in agreement with other studies conducted in dry-ripened "chorizo" [6], being oleic, linoleic, palmitic, stearic and palmitoleic acids the predominated ones in the stages of ripening.

The free fatty acid content during ripening and vacuum packaging showed a gradual and significant release of these compounds as a result of lipolysis. Significant differences $(p<0.05)$ were detected among samples at the end of ripening time. In all cases, CON samples showed lower release values than those obtained for the samples treated with antioxidants. MUFAs were the FFA that showed the highest increases, greater than $70 \%$. Oleic acid was the predominant fatty acid presented in all the batches, with values that ranged between $49 \%$ and 55\%. These values reached the highest levels between days 4 and 19 of ripening, being the samples treated with BHT, TEA $1000, \mathrm{CHE}_{200}$ and BER 1000 that reached the maximum release values. Unlike other authors [32], the percentages of oleic acid continued to increase until the end of ripening and during vacuum storage. 
Table 4. Evolution of free fatty acid composition (mg/100g) of "chorizo" treated with BHT and natural antioxidants during ripening and vacuum-packaging.

\begin{tabular}{|c|c|c|c|c|c|c|c|c|c|c|c|c|c|c|c|c|c|}
\hline & \multirow{2}{*}{ Days } & \multirow{2}{*}{$\mathrm{CON}$} & \multirow{2}{*}{$\begin{array}{c}\text { BHT } \\
200 \\
\end{array}$} & \multicolumn{3}{|c|}{ TEA } & \multicolumn{3}{|c|}{ CHE } & \multicolumn{3}{|c|}{ GRA } & \multicolumn{3}{|c|}{ BER } & \multirow{2}{*}{$\begin{array}{c}p \text { - } \\
\text { Value } \\
\end{array}$} & \multirow{2}{*}{ SEM } \\
\hline & & & & 50 & 200 & 1000 & 50 & 200 & 1000 & 50 & 200 & 1000 & 50 & 200 & 1000 & & \\
\hline \multirow{6}{*}{ C16:0 } & 4 & $274.05^{a, b}$ & $119.86^{\mathrm{a}}$ & $280.66^{\mathrm{a}}$ & $233.36^{\mathrm{a}}$ & $219.68^{a}$ & $207.05^{\mathrm{a}}$ & $247.90^{\mathrm{a}}$ & $159.44^{\mathrm{a}}$ & $250.42^{\mathrm{a}}$ & $215.51^{\mathrm{a}}$ & $223.47^{\mathrm{a}}$ & 244.31 & 194.55 & 167.68 & 0.711 & 13.10 \\
\hline & 19 & $166.96^{\mathrm{a}}$ & $393.41^{\mathrm{b}}$ & $350.09^{\mathrm{a}}$ & $289.73^{\mathrm{a}}$ & $281.74^{\mathrm{a}}$ & $324.98^{\mathrm{a}, \mathrm{b}}$ & $414.83^{a}$ & $227.25^{\text {a }}$ & $227.42^{\mathrm{a}}$ & $210.42^{\mathrm{a}}$ & $303.13^{\mathrm{a}}$ & 271.75 & 288.36 & 297.27 & 0.227 & 16.63 \\
\hline & 48 & $169.33^{a, 1}$ & $353.28^{\mathrm{b}, 3,4}$ & $375.84^{a, 4}$ & $322.48^{a, 2-24}$ & $507.68^{\mathrm{b}, 5}$ & $331.06^{\mathrm{a}, \mathrm{b}, 2-4}$ & $341.90^{\mathrm{a}, 2-2}$ & $396.63^{\mathrm{b}, 4}$ & $317.90^{\mathrm{a}, 2-4}$ & $373.45^{\mathrm{a}, 4}$ & $270.06^{a, 2,3}$ & $330.28^{2-4}$ & $255.06^{2}$ & $314.01^{2-4}$ & 0.000 & 15.12 \\
\hline & 210 & $426.16^{\mathrm{b}}$ & $700.61^{\mathrm{c}}$ & $743.92^{b}$ & $1227.20^{\mathrm{b}}$ & $689.07^{\circ}$ & $801.26^{\mathrm{b}}$ & $936.76^{\mathrm{b}}$ & $820.64^{\circ}$ & $749.68^{\mathrm{b}}$ & $1006.20^{\mathrm{b}}$ & $691.26^{\mathrm{b}}$ & 600.02 & 594.27 & 673.13 & 0.082 & 44.54 \\
\hline & $p$-value & 0.052 & 0.002 & 0.056 & 0.004 & 0.002 & 0.083 & 0.056 & 0.000 & 0.006 & 0.009 & 0.012 & 0.090 & 0.060 & 0.079 & & \\
\hline & SEM & 43.88 & 79.57 & 75.17 & 158.85 & 71.85 & 97.41 & 111.39 & 97.54 & 82.71 & 128.05 & 73.48 & 60.72 & 64.69 & 80.04 & & \\
\hline \multirow{6}{*}{ C16:1 } & 4 & $29.32^{a}$ & $1.64^{\mathrm{a}}$ & $27.99^{\mathrm{a}}$ & $23.72^{\mathrm{a}}$ & $14.56^{\mathrm{a}}$ & $26.28^{a}$ & $26.27^{\mathrm{a}}$ & $11.72^{\mathrm{a}}$ & $26.66^{\mathrm{a}}$ & $13.88^{\mathrm{a}}$ & $16.66^{\mathrm{a}}$ & $18.07^{\mathrm{a}}$ & $17.05^{\mathrm{a}}$ & $8.47^{\mathrm{a}}$ & 0.438 & 2.17 \\
\hline & 19 & $14.16^{\mathrm{a}}$ & $53.91^{\mathrm{b}}$ & $39.91^{\mathrm{a}}$ & $34.66^{\mathrm{a}}$ & $32.77^{\mathrm{a}}$ & $35.07^{\mathrm{a}}$ & $50.31^{\mathrm{a}}$ & $23.83^{\mathrm{a}}$ & $26.46^{\mathrm{a}}$ & $21.55^{\mathrm{a}}$ & $35.22^{\mathrm{a}}$ & $29.97^{\mathrm{a}}$ & $34.88^{\mathrm{a}}$ & $37.55^{\mathrm{a}}$ & 0.081 & 2.39 \\
\hline & 48 & $19.26^{\mathrm{a}, 1}$ & $49.30^{b, 2,3}$ & $54.19^{\mathrm{a}, 3}$ & $43.30^{\mathrm{a}, 2,3}$ & $89.21^{b, 4}$ & $48.80^{\mathrm{a}, 2,3}$ & $43.91^{a, 2,3}$ & $55.45^{\mathrm{b}, 3}$ & $46.49^{\mathrm{a}, 2,3}$ & $57.41^{\mathrm{b}, 3}$ & $38.35^{\mathrm{a}, 2}$ & $47.80^{\mathrm{a}, 2,3}$ & $36.01 \mathrm{a}, 2$ & $43.39^{a, 2,3}$ & 0.000 & 2.95 \\
\hline & 210 & $76.10^{\mathrm{b}, 1}$ & $103.16^{\mathrm{c}, 1,2}$ & $106.19^{\mathrm{b}, 1,2}$ & $167.12^{b, 3}$ & $112.31^{\mathrm{b}, 1,2}$ & $102.71^{b, 1,2}$ & $123.62^{b, 1-3}$ & $109.10^{\mathrm{c}, 1,2}$ & $116.46^{\mathrm{b}, 1,2}$ & $149.79^{\mathrm{c}, 2,3}$ & $97.67^{b, 1}$ & $91.46^{\mathrm{b}, 1}$ & $97.90^{\mathrm{b}, 1}$ & $103.37^{\mathrm{b}, 1,2}$ & 0.038 & 5.09 \\
\hline & $p$-value & 0.009 & 0.003 & 0.010 & 0.001 & 0.001 & 0.006 & 0.030 & 0.000 & 0.013 & 0.000 & 0.009 & 0.019 & 0.032 & 0.026 & & \\
\hline & SEM & 9.60 & 13.85 & 11.72 & 22.18 & 15.27 & 11.56 & 15.07 & 14.33 & 14.60 & 20.50 & 11.92 & 11.11 & 12.45 & 13.90 & & \\
\hline \multirow{6}{*}{ C18:0 } & 4 & 143.67 & $89.44^{\mathrm{a}}$ & 143.01 & $114.11^{\mathrm{a}}$ & $104.97^{\mathrm{a}}$ & 107.01 & 130.30 & $91.68^{\mathrm{a}}$ & $121.10^{\mathrm{a}}$ & $129.85^{\mathrm{a}}$ & $124.49^{\mathrm{a}, \mathrm{b}}$ & 135.63 & 111.36 & 102.55 & 0.900 & 5.87 \\
\hline & 19 & 92.34 & $179.57^{\mathrm{b}}$ & 166.29 & $139.56^{\mathrm{a}}$ & $142.81^{\mathrm{a}, \mathrm{b}}$ & 166.92 & 203.95 & $130.09^{\mathrm{a}}$ & $124.16^{\mathrm{a}}$ & $122.16^{\mathrm{a}}$ & $154.38^{\mathrm{a}, \mathrm{b}}$ & 142.90 & 157.77 & 167.49 & 0.269 & 6.87 \\
\hline & 48 & $69.20^{1}$ & $116.22^{\mathrm{a}, 1,2}$ & $123.21^{2}$ & $96.85^{\mathrm{a}, 1,2}$ & $176.92^{b, 3}$ & $101.52^{1,2}$ & $106.23^{1,2}$ & $133.60^{\mathrm{a}, 2,3}$ & $115.99^{\mathrm{a}, 1,2}$ & $144.12^{\mathrm{a}, 2,3}$ & $104.71^{\mathrm{a}, 1,2}$ & $137.68^{2,3}$ & $99.33^{1,2}$ & $126.71^{2}$ & 0.021 & 5.54 \\
\hline & 210 & 149.17 & $223.33^{\mathrm{b}}$ & 259.89 & $461.58^{\mathrm{b}}$ & $240.33^{c}$ & 345.60 & 351.84 & $280.00^{\mathrm{b}}$ & $232.18^{\mathrm{b}}$ & $395.92^{\mathrm{b}}$ & $245.44^{\mathrm{b}}$ & 215.10 & 211.30 & 321.35 & 0.552 & 22.98 \\
\hline & $p$-value & 0.262 & 0.006 & 0.254 & 0.055 & 0.014 & 0.296 & 0.226 & 0.003 & 0.015 & 0.067 & 0.109 & 0.269 & 0.131 & 0.256 & & \\
\hline & SEM & 16.60 & 20.44 & 25.51 & 62.53 & 19.71 & 49.61 & 45.73 & 27.68 & 19.22 & 48.29 & 23.56 & 16.33 & 19.62 & 41.52 & & \\
\hline
\end{tabular}


Table 4. Cont.

\begin{tabular}{|c|c|c|c|c|c|c|c|c|c|c|c|c|c|c|c|c|c|}
\hline & \multirow{2}{*}{ Days } & \multirow{2}{*}{$\mathrm{CON}$} & \multirow{2}{*}{$\begin{array}{c}\text { BHT } \\
200\end{array}$} & \multicolumn{3}{|c|}{ TEA } & \multicolumn{3}{|c|}{ CHE } & \multicolumn{3}{|c|}{ GRA } & \multicolumn{3}{|c|}{ BER } & \multirow{2}{*}{$\begin{array}{c}p- \\
\text { value }\end{array}$} & \multirow{2}{*}{ SEM } \\
\hline & & & & 50 & 200 & 1000 & 50 & 200 & 1000 & 50 & 200 & 1000 & 50 & 200 & 1000 & & \\
\hline & 4 & $529.15^{\text {a }}$ & $213.02^{a}$ & $468.86^{\mathrm{a}}$ & $443.31^{\mathrm{a}}$ & $338.88^{a}$ & 438.33 & 459.15 & $339.35^{\text {a }}$ & $511.66^{\mathrm{a}}$ & $409.07^{\mathrm{a}}$ & $452.25^{\mathrm{a}}$ & $491.65^{a}$ & $400.20^{\mathrm{a}}$ & $344.88^{\mathrm{a}}$ & 0.465 & 22.37 \\
\hline & 19 & $432.85^{\mathrm{a}, 1}$ & $973.25^{\mathrm{b}, 3,4}$ & $853.91^{\mathrm{a}, 2-4}$ & $733.01^{\mathrm{a}, 1-4}$ & $688.11^{\mathrm{b}, 1-3}$ & $799.28^{2-4}$ & $1043.61^{4}$ & $624.51^{a, b, 1,2}$ & $645.37^{\mathrm{a}, \mathrm{b}, 1,1,2}$ & $551.32^{\mathrm{a}, \mathrm{b}, 1,2}$ & $671.73^{a, 1-3}$ & $682.09^{\mathrm{a}, 1-3}$ & $696.65^{b, 1-3}$ & $784.79^{\mathrm{b}, 2-4}$ & 0.029 & 34.50 \\
\hline C18: & 48 & $405.56^{\mathrm{a}, 1}$ & $1030.46^{\mathrm{b}, 4}$ & $855.82^{\mathrm{a}, 2-4}$ & $875.07^{\mathrm{a}, 2-4}$ & $1278.56^{\mathrm{c}, 5}$ & $858.61^{2-4}$ & $784.46^{2,3}$ & $991.82^{\mathrm{b}, 3,4}$ & $916.95^{\mathrm{b}, 2-4}$ & $1072.72^{b, 4}$ & $803.00^{\mathrm{a}, \mathrm{b}, 2,3}$ & $952.68^{\mathrm{b}, 2-4}$ & $732.70^{\mathrm{b}, 2}$ & $894.76^{b, 2-4}$ & 0.000 & 38.41 \\
\hline \multirow[t]{5}{*}{$\ln 9 c$} & 210 & $899.38^{\mathrm{b}}$ & $1350.71^{\mathrm{c}}$ & $1523.51^{b}$ & $2341.58^{b}$ & $1577.73^{c}$ & 1580.00 & 1791.42 & $1480.70^{c}$ & $1381.90^{\mathrm{c}}$ & $2130.29^{c}$ & $1368.17^{b}$ & $1222.02^{\mathrm{c}}$ & $1306.35^{c}$ & $1378.62^{c}$ & 0.126 & 85.05 \\
\hline & $p$-value & 0.050 & 0.002 & 0.033 & 0.007 & 0.002 & 0.087 & 0.144 & 0.007 & 0.009 & 0.004 & 0.066 & 0.004 & 0.004 & 0.007 & & \\
\hline & SEM & 81.83 & 160.15 & 154.33 & 286.77 & 186.32 & 177.77 & 220.87 & 166.56 & 130.48 & 261.70 & 142.51 & 106.83 & 126.83 & 143.54 & & \\
\hline & 4 & $305.74^{b, 5}$ & $150.08^{a, 1}$ & $270.91^{3-5}$ & $230.20^{\mathrm{a}, 1-5}$ & $186.19^{\mathrm{a}, 1-3}$ & $256.88^{3-5}$ & $273.12^{4,5}$ & $162.99^{\mathrm{a}, 1,2}$ & $226.89^{\mathrm{a}, 1-5}$ & $187.06^{\mathrm{a}, 1-3}$ & $246.44^{2-5}$ & $243.37^{\mathrm{a}, 2-5}$ & $194.95^{\mathrm{a}, 1-4}$ & $185.99^{\mathrm{a}, 1-3}$ & 0.015 & 9.79 \\
\hline & 19 & $257.29^{\mathrm{a}, \mathrm{b}, 1}$ & $397.66^{\mathrm{b}, 3,4}$ & $363.86^{2-4}$ & $351.91^{b, 1-4}$ & $286.12^{\mathrm{a}, \mathrm{b}, \mathrm{l}, \mathrm{2}}$ & $340.63^{1-4}$ & $421.13^{4}$ & $301.49^{\mathrm{b}, 1-3}$ & $326.34^{\mathrm{b}, 1-4}$ & $261.83^{\mathrm{a}, \mathrm{b}, 1}$ & $366.47^{2-4}$ & $373.61^{b, 2-4}$ & $330.43^{b, 1-4}$ & $372.16^{\mathrm{b}, 2-4}$ & 0.038 & 10.72 \\
\hline C18: & 48 & $174.46^{\mathrm{a}, 1}$ & $311.35^{\mathrm{b}, 3,4}$ & $250.16^{2,3}$ & $236.18^{\mathrm{a}, 1-3}$ & $427.53^{b, c, 5}$ & $252.14^{2,3}$ & $205.00^{1,2}$ & $344.28^{\mathrm{b}, \mathrm{c}, 4}$ & $302.38^{\mathrm{a}, \mathrm{b}, 3,34}$ & $339.05^{b, 4}$ & $311.37^{3,4}$ & $352.47^{\mathrm{b}, 4}$ & $308.50^{b, 3,4}$ & $335.79^{b, 4}$ & 0.000 & 13.06 \\
\hline \multirow[t]{5}{*}{$2 n 6 c$} & 210 & $338.14^{\mathrm{b}}$ & $335.41^{\mathrm{b}}$ & 401.34 & $544.78^{\mathrm{c}}$ & $438.75^{\mathrm{c}}$ & 355.56 & 424.95 & $418.13^{\mathrm{c}}$ & $367.91^{\mathrm{b}}$ & $543.81^{\mathrm{c}}$ & 364.37 & $404.98^{\mathrm{b}}$ & $426.23^{\mathrm{c}}$ & $464.93^{\mathrm{b}}$ & 0.299 & 16.58 \\
\hline & p-value & 0.056 & 0.005 & 0.176 & 0.003 & 0.022 & 0.351 & 0.256 & 0.004 & 0.048 & 0.001 & 0.240 & 0.042 & 0.010 & 0.019 & & \\
\hline & SEM & 25.70 & 35.50 & 28.96 & 49.16 & 41.94 & 24.62 & 46.38 & 35.96 & 21.22 & 50.99 & 23.61 & 24.99 & 32.28 & 40.08 & & \\
\hline & 4 & $15.81^{\mathrm{b}, 3}$ & $0.00^{\mathrm{a}, 1}$ & $10.18^{\mathrm{a}, \mathrm{b}, 2}$ & $10.15^{2}$ & $0.00^{\mathrm{a}, 1}$ & $0.00^{1}$ & $9.51^{2}$ & $0.00^{\mathrm{a}, 1}$ & $3.80^{\mathrm{a}, 1}$ & $2.73^{\mathrm{a}, 1}$ & $0.00^{1}$ & $0.00^{\mathrm{a}, 1}$ & $0.00^{\mathrm{a}, 1}$ & $1.72^{\mathrm{a}, 1}$ & 0.000 & 1.03 \\
\hline & 19 & $6.51^{\mathrm{a}}$ & $17.06^{\mathrm{b}}$ & $19.17^{b}$ & 13.94 & $11.99^{\mathrm{a}, \mathrm{b}}$ & 21.82 & 22.62 & $11.58^{\mathrm{b}}$ & $11.91^{\mathrm{b}}$ & $9.77^{\mathrm{a}, \mathrm{b}}$ & 23.90 & $14.21^{b, c}$ & $11.21^{\mathrm{a}, \mathrm{b}}$ & $16.73^{b, c}$ & 0.125 & 1.21 \\
\hline C18: & 48 & $0.00^{\mathrm{a}, 1}$ & 10.98 ab,2 & $5.52^{\mathrm{a}, 1,2}$ & $4.22^{1,2}$ & $20.54^{\mathrm{b}, 3}$ & $7.43^{1,2}$ & $6.13^{1,2}$ & $12.84^{b, 2,3}$ & $8.32^{a, b, 1,2}$ & $11.40^{\mathrm{b}, 2}$ & $8.07^{1,2}$ & $10.55^{\mathrm{b}, 2}$ & $6.10^{\mathrm{a}, 1,2}$ & $10.01^{\mathrm{a}, \mathrm{b}, 2}$ & 0.024 & 1.03 \\
\hline \multirow[t]{5}{*}{$3 n 3$} & 210 & $19.08^{b}$ & $22.13^{b}$ & $21.78^{c}$ & 30.41 & $22.83^{\mathrm{b}}$ & 20.52 & 20.43 & $33.65^{\mathrm{c}}$ & $22.22^{\mathrm{c}}$ & $28.63^{c}$ & 22.37 & $21.06^{\mathrm{c}}$ & $22.97^{b}$ & $22.60^{\mathrm{c}}$ & 0.806 & 1.29 \\
\hline & p-value & 0.009 & 0.055 & 0.184 & 0.001 & 0.054 & 0.161 & 0.217 & 0.001 & 0.009 & 0.002 & 0.092 & 0.006 & 0.026 & 0.022 & & \\
\hline & SEM & 2.96 & 3.44 & 3.05 & 3.72 & 3.73 & 4.16 & 3.32 & 4.64 & 2.66 & 3.66 & 4.30 & 2.96 & 3.40 & 3.11 & & \\
\hline & 4 & $9.54^{\mathrm{a}, \mathrm{b}, 1-3}$ & $0.00^{\mathrm{a}, 1}$ & $17.79^{3}$ & $0.00^{1}$ & $8.36^{\mathrm{a}, 1-3}$ & $0.95^{1}$ & $16.32^{2,3}$ & $0.00^{\mathrm{a}, 1}$ & $0.00^{\mathrm{a}, 1}$ & $2.31^{1}$ & $0.00^{\mathrm{a}, 1}$ & $4.01^{1,2}$ & $0.00^{\mathrm{a}, 1}$ & $0.00^{\mathrm{a}, 1}$ & 0.035 & 1.38 \\
\hline & 19 & $12.65^{\mathrm{a}, \mathrm{b}}$ & $20.26^{b}$ & 15.85 & 14.24 & $9.99^{\mathrm{a}, \mathrm{b}}$ & 15.12 & 19.53 & $8.93^{\mathrm{a}}$ & $14.12^{b}$ & 8.81 & $23.98^{c}$ & 14.91 & $24.90^{c}$ & $18.77^{b, c}$ & 0.247 & 1.25 \\
\hline C20: & 48 & $0.00^{\mathrm{a}}$ & $9.75^{\mathrm{ab}}$ & 16.90 & 7.01 & $29.31^{\mathrm{c}}$ & 9.28 & 9.52 & $6.23^{\mathrm{a}}$ & $9.85^{\mathrm{b}}$ & 8.98 & $12.27^{b}$ & 13.65 & $10.56^{b}$ & $11.27^{\mathrm{a}, \mathrm{b}}$ & 0.081 & 1.48 \\
\hline \multirow[t]{3}{*}{$4 n 6$} & 210 & $21.43^{b}$ & $10.64^{a, b}$ & 13.57 & 15.63 & $19.21^{\mathrm{b}}$ & 7.98 & 7.71 & $23.62^{b}$ & $14.31^{b}$ & 17.65 & $12.72^{b}$ & 19.12 & $23.29^{\mathrm{c}}$ & $28.56^{\mathrm{c}}$ & 0.062 & 1.38 \\
\hline & p-value & 0.167 & 0.084 & 0.989 & 0.068 & 0.012 & 0.126 & 0.254 & 0.026 & 0.003 & 0.232 & 0.019 & 0.082 & 0.000 & 0.035 & & \\
\hline & SEM & 3.50 & 3.07 & 3.63 & 2.64 & 3.30 & 2.23 & 2.35 & 3.49 & 2.24 & 2.61 & 3.39 & 2.36 & 3.85 & 4.26 & & \\
\hline
\end{tabular}

${ }^{a-c}$ Mean values in the same column (same antioxidant in different weeks) with different letter presented significant differences; ${ }^{1-5}$ Mean values in the same row (different antioxidant in the same week) with different number presented significant differences; SEM: standard error of mean; Batches: CON: control; BHT: tert-butyl-4-hydroxytoluene; TEA: tea; CHE: chestnut; GRA: grape seed and BER: beer extracts. 
Table 5. Evolution of main nutritional index $(\mathrm{mg} / 100 \mathrm{~g})$ of "chorizo" treated with BHT and natural antioxidants during ripening and vacuum-packaging.

\begin{tabular}{|c|c|c|c|c|c|c|c|c|c|c|c|c|c|c|c|c|c|}
\hline & \multirow{2}{*}{ Days } & \multirow{2}{*}{$\mathrm{CON}$} & \multirow{2}{*}{$\begin{array}{c}\text { BHT } \\
200 \\
\end{array}$} & \multicolumn{3}{|c|}{ TEA } & \multicolumn{3}{|c|}{ CHE } & \multicolumn{3}{|c|}{ GRA } & \multicolumn{3}{|c|}{ BER } & \multirow{2}{*}{$\begin{array}{c}p \text { - } \\
\text { value }\end{array}$} & \multirow{2}{*}{ SEM } \\
\hline & & & & 50 & 200 & 1000 & 50 & 200 & 1000 & 50 & 200 & 1000 & 50 & 200 & 1000 & & \\
\hline \multirow{6}{*}{$S F A$} & 4 & 417.72 & $209.30^{\mathrm{a}}$ & 423.68 & $347.47^{\mathrm{a}}$ & $324.64^{\mathrm{a}}$ & 314.06 & 378.20 & $251.12^{\mathrm{a}}$ & $371.51^{\mathrm{a}}$ & $345.36^{\mathrm{a}}$ & $347.95^{\text {a }}$ & 379.94 & 305.91 & 270.24 & 0.780 & 18.54 \\
\hline & 19 & 259.29 & $572.99^{\mathrm{b}}$ & 516.38 & $429.29^{\mathrm{a}}$ & $424.54^{\mathrm{a}}$ & 491.91 & 618.78 & $357.33^{b}$ & $351.57^{\mathrm{a}}$ & $332.58^{\mathrm{a}}$ & $457.51^{\mathrm{a}}$ & 414.64 & 446.13 & 464.77 & 0.241 & 23.34 \\
\hline & 48 & $238.52^{1}$ & $469.49^{b, 2-4}$ & $499.04^{3,4}$ & $419.32^{\mathrm{a}, 2-4}$ & $684.60^{\mathrm{b}, 5}$ & $432.58^{2-4}$ & $448.11^{2-4}$ & $530.23^{\mathrm{c}, 4}$ & $433.89^{\mathrm{a}, 2-4}$ & $517.57^{\mathrm{a}, 4}$ & $374.78^{\mathrm{a}, 2,3}$ & $467.96^{2-4}$ & $354.39^{1,2}$ & $440.72^{2-4}$ & 0.001 & 20.17 \\
\hline & 210 & 605.07 & $957.29^{\mathrm{c}}$ & 1039.82 & $1754.88^{b}$ & $962.22^{\mathrm{c}}$ & 1195.06 & 1339.90 & $1152.74^{d}$ & $988.29^{b}$ & $1452.09^{b}$ & $974.31^{\mathrm{b}}$ & 853.55 & 835.24 & 1032.75 & 0.189 & 68.56 \\
\hline & p-value & 0.079 & 0.003 & 0.072 & 0.008 & 0.003 & 0.123 & 0.076 & 0.000 & 0.007 & 0.015 & 0.023 & 0.130 & 0.083 & 0.131 & & \\
\hline & SEM & 62.73 & 103.68 & 103.74 & 229.84 & 95.32 & 152.43 & 162.25 & 132.27 & 102.49 & 183.00 & 102.29 & 84.44 & 89.03 & 127.86 & & \\
\hline \multirow{6}{*}{ MUFA } & 4 & $560.24^{\mathrm{a}}$ & $214.66^{\mathrm{a}}$ & $496.85^{\mathrm{a}}$ & $467.03^{a}$ & $353.43^{\mathrm{a}}$ & 464.60 & 485.42 & $351.06^{\mathrm{a}}$ & $538.32^{\mathrm{a}}$ & $422.95^{\mathrm{a}}$ & $468.91^{\mathrm{a}}$ & $509.72^{\mathrm{a}}$ & $417.25^{\mathrm{a}}$ & $353.35^{\mathrm{a}}$ & 0.432 & 24.10 \\
\hline & 19 & $447.01^{\mathrm{a}, 1}$ & $1027.17^{\mathrm{b}, 3,4}$ & $893.82^{a, 2-4}$ & $767.66^{\mathrm{a}, 1-4}$ & $720.87^{b, 1-3}$ & $834.35^{2-4}$ & $1093.92^{4}$ & $648.35^{\mathrm{a}, \mathrm{b}, \mathrm{l}, \mathrm{2}}$ & $671.83^{\mathrm{a}, \mathrm{b}, 1,2}$ & $572.87^{\mathrm{a}, \mathrm{b}, 1,1,2}$ & $706.95^{\mathrm{a}, 1-3}$ & $712.06^{\mathrm{a}, 1-3}$ & $731.53^{\mathrm{a}, \mathrm{a}, \mathrm{l},-3}$ & $822.34^{b, 2-4}$ & 0.031 & 36.77 \\
\hline & 48 & $424.81^{\mathrm{a}, 1}$ & $1079.76^{\mathrm{b}, 4}$ & $910.00^{\mathrm{a}, 2-4}$ & $918.37^{\mathrm{a}, 2-4}$ & $1368.99^{\mathrm{c}, 5}$ & $907.41^{2-4}$ & $828.37^{2,3}$ & $1047.27^{b, 3,4}$ & $963.44^{b, 2-4}$ & $1130.13^{b, 4}$ & $841.36^{\mathrm{a}, \mathrm{b}, 2,3}$ & $1000.48^{b, 2-4}$ & $768.70^{\mathrm{b}, 2}$ & $938.15^{b, 2-4}$ & 0.000 & 41.11 \\
\hline & 210 & $990.17^{b}$ & $1469.62^{c}$ & $1650.85^{\mathrm{b}}$ & $2546.22^{b}$ & $1712.08^{c}$ & 1709.73 & 1941.17 & $1608.25^{c}$ & $1522.29^{c}$ & $2313.74^{c}$ & $1481.89^{b}$ & $1327.59^{c}$ & $1419.73^{c}$ & $1498.93^{c}$ & 0.112 & 91.21 \\
\hline & p-value & 0.039 & 0.001 & 0.028 & 0.006 & 0.001 & 0.077 & 0.133 & 0.005 & 0.008 & 0.003 & 0.057 & 0.004 & 0.005 & 0.008 & & \\
\hline & SEM & 93.32 & 174.94 & 168.48 & 314.60 & 204.09 & 193.11 & 239.88 & 183.01 & 148.03 & 287.04 & 156.57 & 119.33 & 141.34 & 159.35 & & \\
\hline \multirow{6}{*}{ PUFA } & 4 & $331.08^{b, 5}$ & $150.08^{\mathrm{a} .1}$ & $298.87^{4,5}$ & $240.35^{\mathrm{a}, 1-5}$ & $194.55^{\mathrm{a}, 1-3}$ & $257.83^{3-5}$ & $298.93^{4,5}$ & $162.99^{\mathrm{a}, 1,2}$ & $230.68^{\mathrm{a}, 1-4}$ & $192.10^{\mathrm{a}, 1-3}$ & $246.44^{2-5}$ & $247.38^{\mathrm{a}, 2-5}$ & $194.95^{\mathrm{a}, 1-3}$ & $187.72^{\mathrm{a}, 1-3}$ & 0.008 & 11.29 \\
\hline & 19 & $276.43^{\mathrm{a}, \mathrm{b}, 1}$ & $434.97^{\mathrm{c}, 4,5}$ & $398.87^{1-5}$ & $380.08^{b, 1-5}$ & $308.09^{\mathrm{a}, 1-3}$ & $377.57^{1-5}$ & $463.27^{5}$ & $321.99^{b, 1-4}$ & $352.37^{\mathrm{b}, 1-5}$ & $280.42^{b, 1,2}$ & $414.35^{3-5}$ & $402.73^{b, 2-5}$ & $366.53^{b, 1-5}$ & $407.65^{b, c, 3-5}$ & 0.050 & 12.55 \\
\hline & 48 & $174.46^{\mathrm{a}, 1}$ & $332.07^{b, 3,4}$ & $272.57^{2,3}$ & $247.41^{\mathrm{a}, 1-3}$ & $477.38^{\mathrm{b}, 5}$ & $268.84^{2,3}$ & $220.64^{1,2}$ & $363.35^{\mathrm{b}, 4}$ & $320.54^{\mathrm{a}, \mathrm{b}, 3,4}$ & $359.42^{\mathrm{b}, 4}$ & $331.71^{3,4}$ & $376.66^{\mathrm{b}, 4}$ & $325.15^{b, 3,4}$ & $357.07^{\mathrm{b}, 4}$ & 0.000 & 14.81 \\
\hline & 210 & $386.20^{\mathrm{b}}$ & $372.15^{b, c}$ & 444.05 & $601.60^{\mathrm{c}}$ & $490.66^{b}$ & 394.38 & 460.58 & $480.62^{\mathrm{c}}$ & $414.98^{b}$ & $601.65^{\circ}$ & 403.06 & $451.71^{\mathrm{b}}$ & $479.32^{c}$ & $522.59^{\mathrm{c}}$ & 0.348 & 18.41 \\
\hline & p-value & 0.050 & 0.005 & 0.226 & 0.002 & 0.019 & 0.284 & 0.256 & 0.002 & 0.030 & 0.001 & 0.169 & 0.030 & 0.008 & 0.018 & & \\
\hline & SEM & 32.42 & 41.13 & 33.59 & 56.28 & 49.13 & 30.73 & 51.10 & 43.67 & 26.91 & 58.12 & 30.73 & 30.60 & 39.74 & 48.02 & & \\
\hline
\end{tabular}


Table 5. Cont.

\begin{tabular}{|c|c|c|c|c|c|c|c|c|c|c|c|c|c|c|c|c|c|}
\hline & \multirow{2}{*}{ Days } & \multirow{2}{*}{ CON } & \multirow{2}{*}{$\begin{array}{c}\text { BHT } \\
200 \\
\end{array}$} & \multicolumn{3}{|c|}{ TEA } & \multicolumn{3}{|c|}{ CHE } & \multicolumn{3}{|c|}{ GRA } & \multicolumn{3}{|c|}{ BER } & \multirow{2}{*}{$\begin{array}{c}p- \\
\text { value }\end{array}$} & \multirow{2}{*}{ SEM } \\
\hline & & & & 50 & 200 & 1000 & 50 & 200 & 1000 & 50 & 200 & 1000 & 50 & 200 & 1000 & & \\
\hline \multirow{6}{*}{$P / S$} & 4 & 0.88 & $0.72^{\mathrm{b}}$ & 0.74 & $0.72^{b, c}$ & 0.62 & 0.86 & $0.83^{b}$ & $0.65^{\mathrm{b}}$ & 0.68 & 0.56 & $0.72^{\mathrm{a}, \mathrm{b}}$ & $0.66^{\mathrm{a}}$ & 0.66 & 0.72 & 0.961 & 0.03 \\
\hline & 19 & 1.07 & $0.77^{\mathrm{b}}$ & 0.83 & $0.90^{\circ}$ & 0.73 & 0.78 & $0.76^{\mathrm{b}}$ & $0.91^{\mathrm{c}}$ & 1.02 & 0.91 & $0.91^{\mathrm{b}}$ & $0.98^{\mathrm{b}}$ & 0.83 & 0.91 & 0.597 & 0.03 \\
\hline & 48 & $0.78^{2-5}$ & $0.72^{b, 1-5}$ & $0.55^{1,2}$ & $0.60^{\mathrm{a}, \mathrm{b}, 1-3}$ & $0.70^{1-5}$ & $0.62^{1-3}$ & $0.50^{\mathrm{a}, \mathrm{b}, \mathrm{l}}$ & $0.69^{b, 1-4}$ & $0.74^{2-5}$ & $0.71^{1-5}$ & $0.89^{\mathrm{b}, 4,5}$ & $0.81^{\mathrm{a}, \mathrm{b}, 3-5}$ & $0.92^{5}$ & $0.82^{3-5}$ & 0.015 & 0.03 \\
\hline & 210 & $0.64^{5}$ & $0.39^{\mathrm{a}, 1-3}$ & $0.43^{1-4}$ & $0.35^{\mathrm{a}, 1,2}$ & $0.51^{1-5}$ & $0.35^{1,2}$ & $0.34^{\mathrm{a}, 1}$ & $0.42^{\mathrm{a}, 1-4}$ & $0.42^{1-4}$ & $0.43^{1-4}$ & $0.42^{\mathrm{a}, 1-4}$ & $0.56^{\mathrm{a}, 3-5}$ & $0.60^{4,5}$ & $0.54^{2-5}$ & 0.036 & 0.02 \\
\hline & p-value & 0.489 & 0.004 & 0.140 & 0.016 & 0.158 & 0.203 & 0.052 & 0.005 & 0.084 & 0.164 & 0.044 & 0.056 & 0.163 & 0.346 & & \\
\hline & SEM & 0.09 & 0.06 & 0.07 & 0.08 & 0.04 & 0.09 & 0.08 & 0.07 & 0.09 & 0.08 & 0.08 & 0.07 & 0.06 & 0.07 & & \\
\hline \multirow{6}{*}{$n 6 / n 3$} & 4 & $20.25^{\mathrm{b}, 2}$ & $0.00^{\mathrm{a}, 1}$ & $30.45^{2,3}$ & $22.72^{b, 2}$ & $0.00^{\mathrm{a}, 1}$ & $0.00^{1}$ & $37.77^{2,3}$ & $0.00^{\mathrm{a}, 1}$ & $59.71^{\mathrm{b}, 4}$ & $46.83^{b, 3,4}$ & $0.00^{\mathrm{a}, 1}$ & $0.00^{\mathrm{a}, 1}$ & $0.00^{\mathrm{a}, 1}$ & $0.00^{\mathrm{a}, 1}$ & 0.000 & 4.00 \\
\hline & 19 & $41.49^{\mathrm{c}, 4}$ & $24.72^{b, 1-3}$ & $21.80^{1-3}$ & $26.23^{c, 1-3}$ & $25.29^{b, 1-3}$ & $19.39^{1,2}$ & $19.59^{1,2}$ & $26.85^{\mathrm{c}, 1-3}$ & $28.58^{a, 2,3}$ & $29.09^{a, 2,3}$ & $16.96^{\mathrm{b}, 1}$ & $27.36^{\mathrm{c}, 1-3}$ & $31.85^{\mathrm{b}, 3,4}$ & $23.66^{b, 1-3}$ & 0.011 & 1.29 \\
\hline & 48 & $0.00^{\mathrm{a}}$ & $29.71^{\mathrm{b}}$ & 48.95 & $41.03^{d}$ & $24.48^{b}$ & 38.01 & 45.20 & $29.04^{c}$ & $43.73^{a, b}$ & $31.36^{\mathrm{a}}$ & $40.16^{c}$ & $34.75^{\mathrm{d}}$ & $53.04^{c}$ & $35.76^{b}$ & 0.072 & 2.92 \\
\hline & 210 & $18.85^{b}$ & $17.29^{b}$ & 19.50 & $18.46^{\mathrm{a}}$ & $20.21^{b}$ & 19.06 & 22.21 & $13.16^{b}$ & $17.23^{\mathrm{a}}$ & $19.61^{\mathrm{a}}$ & $19.28^{b}$ & $20.50^{b}$ & $20.56^{b}$ & $22.05^{b}$ & 0.725 & 0.67 \\
\hline & $p$-value & 0.000 & 0.014 & 0.105 & 0.000 & 0.018 & 0.066 & 0.578 & 0.007 & 0.048 & 0.027 & 0.005 & 0.000 & 0.001 & 0.009 & & \\
\hline & SEM & 5.56 & 4.46 & 5.05 & 3.22 & 4.10 & 5.66 & 6.73 & 4.56 & 6.61 & 3.94 & 5.53 & 4.92 & 7.34 & 5.06 & & \\
\hline
\end{tabular}

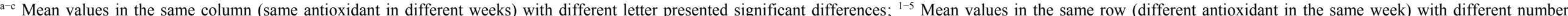
presented significant differences; SEM: standard error of mean; Batches: CON: control; BHT: tert-butyl-4-hydroxytoluene; TEA: tea; CHE: chestnut; GRA: grape seed and BER: beer extracts. 
The increases were lower in PUFA and SFA, with values between $3 \%-145 \%$ and $8 \%-111 \%$, respectively. Regarding PUFA, linoleic and arachidonic were the fatty acids that showed higher percentages of release. The samples treated with $\mathrm{CHE}_{200}$ showed a decrease (26\%) during ripening that could be associated with the oxidation of PUFA and the decrease in the proportion of the long chain PUFAs such as arachidonic acid (42\%) [33]. Within SFA, palmitic and stearic acid were the most abundant, with levels between $17.4 \%-22.8 \%$ and $6.2 \%-8.1 \%$, respectively. Unlike what happened with oleic acid, stearic decreased toward the end of ripening in $\mathrm{CON}$ and in the samples treated with low dose of natural extracts (mean decreases of $52 \%$ vs. $12 \%$ ), for increased again until the end of vacuum packaged.

The amount of PUFA can be used as a measurement of the oxidative deterioration of meats, due to containing double bonds in the hydrocarbon chain being preferred substrates in oxidative reactions. Regarding oxidative stability, a significant correlation was found between PUFA and TBARS $(r=-0.22, p<0.05)$. As can be seen in Figure 3, the oxidative degradation of PUFA mainly occurred after day 19. At the end of ripening, we observed that the addition of GRA 1000 and $\mathrm{BER}_{200}$ extracts protect chorizos from oxidative degradation since, higher amount of PUFA were observed in these treated samples than in $\mathrm{CON}(21.5 \%$ and $22.4 \%$ vs. $21.1 \%$, respectively).
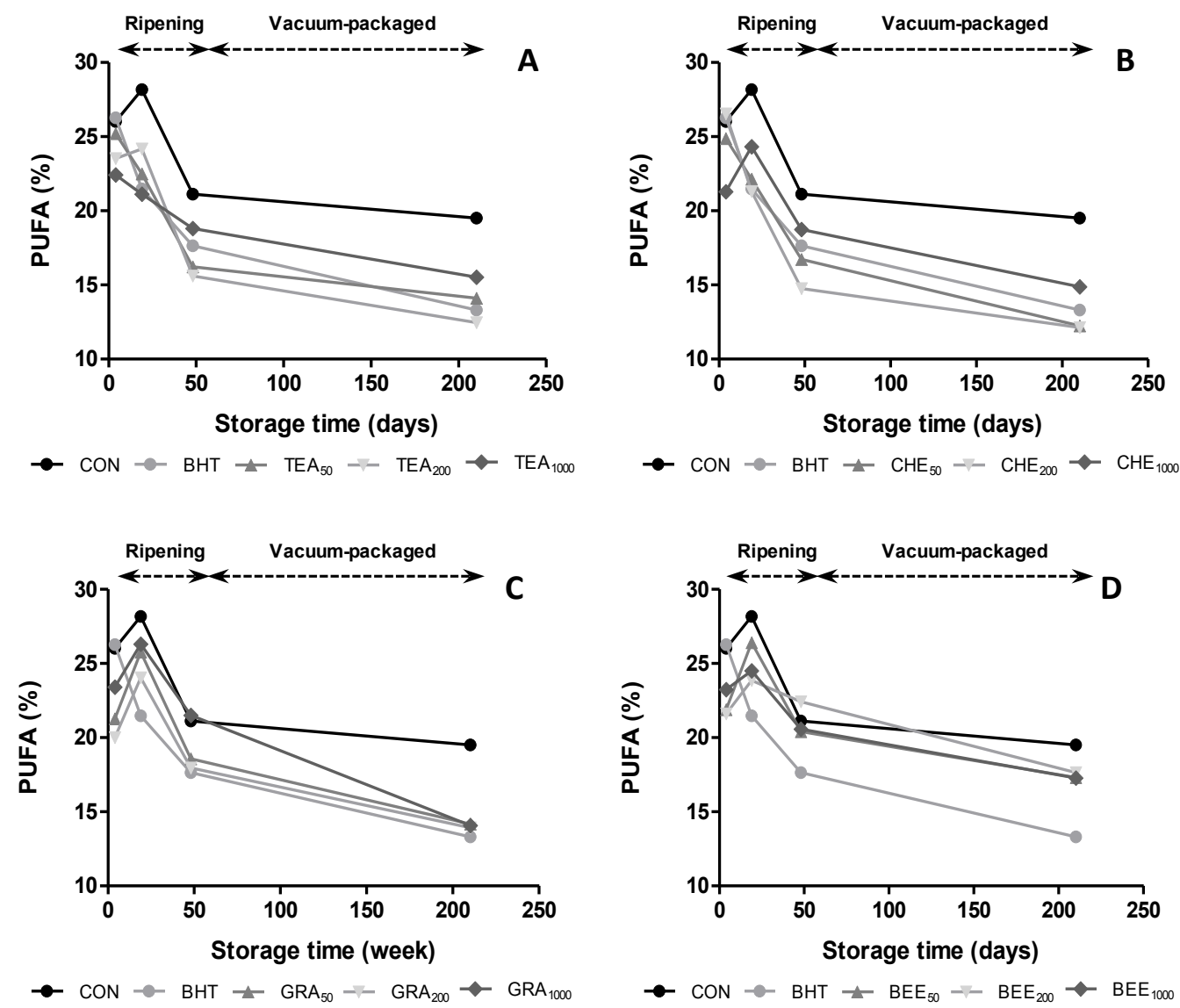

Figure 3. Evolution of PUFA content in dry-cures sausages treated with BHT and natural antioxidants during ripening and vacuum-packaged storage.

To assess the nutritional properties of IMF, the ratios PUFA/SFA and $n-6 / n-3$ were determined (Table 5). The PUFA/SFA ratio showed mean values of 0.72 , being $\mathrm{CON}$ and the samples treated with 
BER extract which showed the highest values. Significant differences $(p<0.05)$ were found among samples at the end of ripening and within storage time in samples treated with BHT and with higher doses of natural antioxidants $\left(\mathrm{TEA}_{200}, \mathrm{CHE}_{200}, \mathrm{CHE}_{1000}\right.$ and $\left.\mathrm{GRA}_{1000}\right)$. In general, at the end of ripening the obtained values were within the typical values (0.5-0.7) of the Mediterranean diet [34] and lower than the FAO recommendations [35] for human diet (0.85), while at 210 days the values were lower (mean values lower than 0.46).

\section{Conclusions}

The addition of natural antioxidants changed the physicochemical properties of dry-cured sausages. The presence of antioxidants and the use of low concentrations improved color maintenance during ripening and under vacuum conditions. The results obtained for TBARS values showed that natural antioxidants matched or even improved the results obtained for BHT, with higher effectiveness for grape and chestnut extracts. The values of hardness decreased significant with the addition of antioxidants, obtaining the lowest values with the intermediate dose. Microbial counts were affected by the addition of antioxidants since lower counts were observed in sausages prepared with natural extracts. Free fatty acid content during ripening and vacuum packaging showed a gradual and significant release. The addition of grape and beer extracts protected sausages from oxidative degradation. Further analysis of how to affect the addition of natural extracts on sensory properties and volatile compounds of chorizo will be addressed.

\section{Acknowledgment}

Authors are grateful to Galician government (Xunta de Galicia 09TAL006CT) for the financial support.

\section{Author Contributions}

Mirian Pateiro and Roberto Bermúdez have performed the measurements and analyzed the data. Jose Manuel Lorenzo and Daniel Franco have designed and supervised the research. All authors have contributed to the writing of the manuscript and have approved the final paper.

\section{Conflicts of Interest}

The authors declare no conflict of interest.

\section{References}

1. O’Grady, M.N.; Carpenter, R.; Lynch, P.B.; O’Brien, N.M.; Kerry, J.P. Addition of grape seed extract and bearberry to porcine diets: Influence on quality attributes of raw and cooked pork. Meat Sci. 2008, 78, 438-446.

2. Jayaprakasha, G.K.; Selvi, T.; Sakariah, K.K. Antibacterial and antioxidant activities of grape (Vitis vinifera) seed extracts. Food Res. Int. 2003, 36, 117-122.

3. Lorenzo, J.M.; Sineiro, J.; Amado, I.R.; Franco, D. Influence of natural extracts on the shelf life of modified atmosphere-packaged pork patties. Meat Sci. 2014, 96, 526-534. 
4. Pateiro, M.; Lorenzo, J.M.; Amado, I.R.; Franco, D. Effect of addition of green tea, chestnut and grape extract on the shelf-life of pig liver pâté. Food Chem. 2014, 147, 386-394.

5. Lorenzo, J.M.; González-Rodríguez, R.M.; Sánchez, M.; Amado, I.R.; Franco, D. Effects of natural (grape seed and chestnut extract) and synthetic antioxidants (buthylatedhydroxytoluene, BHT) on the physical, chemical, microbiological and sensory characteristics of dry cured sausage “chorizo". Food Res. Int. 2013, 54, 611-620.

6. Gómez, M.; Lorenzo, J.M. Effect of fat level on physicochemical, volatile compounds and sensory characteristics of dry-ripened "chorizo" from Celta pig breed. Meat Sci. 2013, 95, 658-666.

7. Moure, A.; Cruz, J.M.; Franco, D.; Domínguez, J.M.; Sineiro, J.; Domínguez, H.; Núñez, M.J.; Parajó, J.C. Natural antioxidants from residual sources. Food Chem. 2001, 72, 145-171.

8. Summo, C.; Caponio, F.; Paradiso, V.M.; Pasqualone, A.; Gomes, T. Vacuum-packed ripened sausages: Evolution of oxidative and hydrolytic degradation of lipid fraction during long-term storage and influence on the sensory properties. Meat Sci. 2010, 84, 147-151.

9. Nassu, R.T.; Gonçalves, L.A.G.; da Silva, M.A.A.P.; Beserra, F.J. Oxidative stability of fermented goat meat sausage with different levels of natural antioxidant. Meat Sci. 2003, 63, 43-49.

10. Tang, S.Z.; Ou, S.Y.; Huang, X.S.; Li, W.; Kerry, J.P.; Buckley, D.J. Effects of added tea catechins on colour stability and lipid oxidation in minced beef patties held under aerobic and modified atmospheric packaging conditions. J. Food Eng. 2006, 77, 248-253.

11. Marco, G.J. A rapid method for evaluation of antioxidants. J. Am. Oil Chem. Soc. 1968, 45, 594-598.

12. ISO (International Organization for Standardization). Determination of moisture content, ISO 1442:1997 standard. In International Standards of Meat and Meat Products; ISO: Genève, Switzerland, 1997.

13. Targladis, B.G.; Watts, B.M.; Younathan, M.T.; Duggan, L.R. A distillation method for the quantitative determination of malonaldehyde in rancid foods. J. Am. Oil Chem. Soc. 1960, 37, 44-48.

14. Folch, J.; Lees, M.; Sloane-Stanley, G.H. A simple method for the isolation and purification of total lipids from animal tissues. J. Biol. Chem. 1957, 226, 497-509.

15. García-Regueiro, J.A.; Gilbert, J.; Díaz, I. Determination of neutral lipids from subcutaneous fat cured ham by capillary gas chromatography and liquid chromatography. J. Chromatogr. A 1994, $667,225-233$.

16. Carreau, J.P.; Dubacq, J.P. Adaptation of a macro-scale method to the micro-scale for fatty acid methyl transesterification of biological lipid extracts. J. Chromatogr. A 1978, 151, 384-390.

17. Lorenzo, J.M.; Franco, D. Fat effect on physico-chemical, microbial and textural changes through the manufactured of dry-cured foal sausage. Lipolysis, proteolysis and sensory properties. Meat Sci. 2012, 92, 704-714.

18. Rubilar, M.; Pinelo, M.; Shene, C.; Sineiro, J.; Nunez, M.J. Separation and HPLC-MS identification of phenolic antioxidants from agricultural residues: Almond hulls and grape pomace. J. Agric. Food Chem. 2007, 55, 10101-10109. 
19. Van der Hooft, J.J.; Akermi, M.; Unlu, F.M.; Mihaleva, V.; Gomez-Roldan, V.; Bino, R.J.; de Vos, R.C.H.; Vervoort, J. Structural annotation and elucidation of conjugated phenolic compounds in black, green, and white tea extracts. J. Agric. Food Chem. 2012, 60, 8841-8850.

20. Fontecave, M.; Lepoivre, M.; Elleingand, E.; Gerez, C.; Guittet, O. Resveratrol, a remarkable inhibitor of ribonucleotide reductase. FEBS Lett. 1998, 421, 277-279.

21. Lorenzo, J.M.; Gómez, M.; Fonseca, S. Effect of commercial starter cultures on physicochemical characteristics, microbial counts and free fatty acid composition of dry-cured foal sausage. Food Control 2014, 46, 382-389.

22. Lorenzo, J.M.; Michinel, M.; López, M.; Carballo, J. Biochemical characteristics of two Spanish traditional dry-cured sausage varieties: Androlla and Botillo. J. Food Compos. Anal. 2000, 13, 809-817.

23. Lorenzo, J.M.; Temperán, S.; Bermúdez, R.; Cobas, N.; Purriños, L. Changes in physico-chemical, microbiological, textural and sensory attributes during ripening of dry-cured foal salchichón. Meat Sci. 2012, 90, 194-198.

24. Liaros, N.G.; Katsanidis, E.; Bloukas, J.G. Effect of the ripening time under vacuum and packaging film permeability on processing and quality characteristics of low-fat fermented sausages. Meat Sci. 2009, 83, 589-598.

25. Jayawardana, B.C.; Hirano, T.; Han, K.; Ishii, H.; Okada, T.; Shibayama, S.; Fukushima, M.; Sekikawa, M.; Shimada, K. Utilization of adzuki bean extract as a natural antioxidante in cured and uncured cooked pork sausages. Meat Sci. 2011, 89, 150-153.

26. González-Fernández, C.; Santos, E.M.; Rovira, J.; Jaime, I. The effect of sugar concentration and starter culture on instrumental and sensory textural properties of "chorizo", Spanish dry-cured sausage. Meat Sci. 2006, 74, 467-475.

27. Jongberg, S.; Gislason, N.E.; Lund, M.N.; Skibsted, L.H.; Waterhouse, A.L. Thiol-quinone adduct formation in myofibrillar proteins detected by LC-MS. J. Agric. Food Chem. 2011, 59, 6900-6905.

28. Jongberg, S.; Tørngren, M.A.; Gunvig, A.; Skibsted, L.H.; Lund, M.N. Effect of green tea or rosemary extract on protein oxidation in Bologna type sausages prepared from oxidatively stressed pork. Meat Sci. 2013, 93, 538-546.

29. Bozkurt, H.; Bayram, M. Colour and textural attributes of sucuk during ripening. Meat Sci. 2006, 73, 344-350.

30. Coşkuner, O.; Ertaş, A.H.; Soyer, A. The effect of processing method and storage time on constituents of Turkish sausages (sucuk). J. Food Process. Preserv. 2010, 34, 125-135.

31. Greene, B.E.; Cumuze, T.H. Relationship between TBA numbers and inexperienced panelist's assessments of oxidized-avor in cooked beef. J. Food Sci. 1982, 47, 52-58.

32. Hierro, E.; de la Hoz, L.; Ordóñez, J.A. Contribution of microbial and meat endogenous enzymes to the lipolysis of dry fermented sausages. J. Agric. Food Chem. 1997, 45, 2989-2995.

33. Qiu, C.; Zhao, M.; Sun, W.; Zhou, F.; Cui, C. Changes in lipid composition, fatty acid profile and lipid oxidative stability during Cantonese sausage processing. Meat Sci. 2013, 93, 525-532.

34. Ulbricht, T.L.V.; Southgate, D.A.T. Coronary heart disease: Seven dietary factors. Lancet 1991, $338,985-992$. 
35. FAO (Food and Agriculture Organization of the United Nations). Fat and fatty acid requirements for adults. In Fats and Fatty Acids in Human Nutrition; FAO: Rome, Italy, 2010; pp. 55-62.

(C) 2015 by the authors; licensee MDPI, Basel, Switzerland. This article is an open access article distributed under the terms and conditions of the Creative Commons Attribution license (http://creativecommons.org/licenses/by/4.0/). 